\title{
Sliding Dynamics of a Filippov Forest-Pest Model with Threshold Policy Control
}

\author{
Lirong Liu $\mathbb{D}^{D}$, Changcheng Xiang $\mathbb{D}^{D}$, Guangyao Tang, and Yuan Fu \\ School of Science, Hubei Minzu University, Enshi, Hubei 445000, China \\ Correspondence should be addressed to Changcheng Xiang; xcc7426681@126.com
}

Received 6 August 2019; Revised 1 October 2019; Accepted 21 October 2019; Published 11 November 2019

Academic Editor: Xianggui Guo

Copyright (c) 2019 Lirong Liu et al. This is an open access article distributed under the Creative Commons Attribution License, which permits unrestricted use, distribution, and reproduction in any medium, provided the original work is properly cited.

\begin{abstract}
A novel Filippov forest-pest system with threshold policy control (TPC) is established while an economic threshold (ET) is used to guide switching. The aim of our work is to address how to reasonably and successfully control pests by means of sliding dynamics for the Filippov system. On the basis of the above considerations, conditions for the existence and stability of equilibria of subsystems are addressed, and the sliding segments and several types of equilibria of the proposed system are defined. These equilibria include the regular/virtual equilibrium, pseudoequilibrium, boundary equilibrium, and tangent point. Further, not only are the relations between nullclines and equilibria of the Filippov system discussed, but the relations between pseudoequilibrium, nullclines, and the sliding segment are discussed. More importantly, four cases of sliding bifurcations of the Filippov system with respect to different types of equilibria of subsystems are investigated, and the corresponding biological implications concerning integrated pest management (IPM) are analyzed. Our results show that the points of intersection between nullclines are equilibria of the system, and the two endpoints of the sliding segment are on the nullclines. It is also verified that the pseudoequilibrium is the point of intersection of the sliding segment and nullclines of the Filippov system, and the pseudoequilibrium exists on the sliding segment. More interestingly, sliding dynamics analysis reveals that the Filippov system has sliding limit cycles, a bistable state and a stable refuge equilibrium point, and the optimal time and strategy for controlling pests are provided.
\end{abstract}

\section{Introduction}

Because insect infestations increase forest mortality, which in turn affects the carbon cycle and causes air pollution [1], many scholars have paid much attention to the effects of disturbances on forests [2-7]. Disturbances are classified as natural and anthropogenic disturbances. Natural disturbances include wildfires, insect infestations, floods, droughts, and bad weather while anthropogenic disturbances include deforestation, the logging of timber, and the spraying of pesticides [4]. Hence, it is vital that the effects of disturbances on the forest are solved. To do this, the age structures of trees in forests where pests exist have been investigated, and strategies for forest management and fire protection have been developed by considering the size of fires, fire control methods, and the behaviors of planted trees [6-10]. However, few researchers have addressed the insect infestations from a biological point of view.
The dynamics of numerous real-world systems can be modeled using discontinuous differential equations [11-15]. As an example, Filippov pest control models have been proposed to investigate sliding bifurcations [16]. Yang and Liao studied the Filippov Hindmarsh-Rose neuron model for several sliding bifurcation phenomena, and the threshold policy control (TPC) was considered [17]. Wang et al. proposed a Filippov epidemic model in discussing the effects of factors on controlling epidemic diseases [18]. Moreover, research on the related Filippov system has focused on bifurcation analysis [16,19-22]. Tan et al. discussed the sliding bifurcation analysis for a Filippov predator-prey system [23]. $\mathrm{Qu}$ and $\mathrm{Li}$ investigated the sliding phenomenon in Filippov dynamical systems based on Chua's circuit [24]. Therefore, combining integrated pest management (IPM), a Filippov system with TPC (i.e., a discontinuous piecewise smooth system [25]) is established to reasonably and successfully control pests. It is a challenging but important work to determine the functional response of pests [26-28]. 
IPM is a well-known strategy of controlling pests effectively [29-35], which includes biological strategies (i.e., releasing natural enemies), cultural strategies (i.e., acquiring or capturing pests artificially), and chemical strategies (i.e., spraying a certain dosage of pesticides) that are used for pest control to avoid exceeding an economic threshold (ET) [36]. In other words, the three types of strategies aim to reduce the number of pests below the economic injury level (EIL), i.e., the environment is not destroyed [37]. TPC, a control strategy, is implemented once the number of pests reaches and exceeds the ET [38-42].

Recently, Xiao and Bosch have built a model with respect to the effects of pest on biologically based technologies for controlling pest (BBTs), which revealed that biological control has two effects including stabilization and destabilization [43]. Stern et al. have verified that integrated control was the most effective where chemical treatment was carried out for successful pest control and pest eradication was not necessary to be considered, and an ET was applied to determine the use of pesticides [36]. Liang and Tang have analyzed the key factors (i.e., optimum timing, the dosage of pesticide, and ET) which affect pest management by employing different impulsive differential equations [44]. A pest-natural enemy system has been proposed by Liang et al., and pesticide resistance was taken into account, which aimed to determine the number of releasing natural enemies to ensure pest eradication [45]. The present paper focuses on the control of pests (beetles), and the sliding dynamics of a Filippov system based on the tree-beetle model is discussed.

First, a novel Filippov forest-pest model is proposed by combining IPM and TPC. Then, conditions for the existence and stability of equilibria of subsystems are addressed, which is helpful to study the dynamics of the proposed system. Further, the sliding segments and several types of equilibria of the system are defined, and these equilibria include the regular/virtual equilibrium, pseudoequilibrium, boundary equilibrium, and tangent point. The relations between nullclines and equilibria of the Filippov system are discussed, which reveal that the points of intersection between nullclines are these equilibrium points of the system. Moreover, the relations between pseudoequilibrium, nullclines and the sliding segment are also discussed. The results verify that pseudoequilibrium is the point of intersection of the sliding segment and nullclines of the Filippov system, and the pseudoequilibrium exists on the sliding segment.

The sliding bifurcations of the Filippov system are addressed for different values of ET. Results show that the value of ET affects the control range and also the time that we decide to take action to prevent the density of the pest population from reaching the value of ET. Therefore, it is important to choose the optimal strategy when the pest density reaches the ET. More importantly, the Filippov system has sliding limit cycles, a bistable state, and a stable refuge equilibrium point (i.e., when the pest population with relatively small intrinsic growth rate, it will be stable at the small density).
The remainder of the paper is organized as follows. In Section 2, the model description and preliminaries of the Filippov system are introduced. In Section 3, equilibria and sliding dynamics including the conditions for the existence and stability of equilibria of subsystems are addressed. Moreover, the sliding regions and equilibria of the Filippov system are discussed. In Section 4, the sliding bifurcations of the Filippov system with respect to different types of equilibria of subsystems are presented, and the corresponding biological implications related to pest control are analyzed. Conclusions drawn from the results of our work are presented in Section 5.

\section{Model Description and Preliminaries}

2.1. Model Description. To explore the effects of insect infestations on the forest, the classical tree-beetle model was considered [4]:

$$
\left\{\begin{array}{l}
\dot{V}(t)=r_{v} V(t)\left(1-\frac{V(t)}{k_{v}}-m(B)\right) \\
\dot{B}(t)=r_{b} B(t)\left(1-\frac{B(t)}{k_{b}}\right)-\frac{\alpha B^{2}(t)}{1+\beta B^{2}(t)}
\end{array}\right.
$$

where the number of susceptible trees is denoted by $V(t)$, $B(t)$ represents the population density of mountain pine beetles per tree, $r_{v}$ and $r_{b}$ are the intrinsic growth rates of $V(t)$ and $B(t)$, respectively, and their carrying capacity is denoted by $k_{v}$ and $k_{b}$, respectively, $\alpha$ is a positive constant that depends upon trees, and $\beta$ is also a positive constant that determines the scale at which beetle density generates saturation.

Note that the beetles can be treated as herbivore type insects, which consume trees [46-48]. The density growth of trees is subject to a logistic self-interaction in the absence of beetles where the forest does not have any defense against insect predation. In particular, a functional response (i.e., the Holling type-III functional response) is assumed [26]. The functional response of the predator is an important part of the prey-predator relationship, which is the impact of the predator (pest) per unit time on the change in prey (forest) density. Types of functional response include Holling types I-III $[26,49]$, the ratio-dependence type [27, 50], and the Hassell-Varley type [28].

When the beetles are introduced in the first equation of model (1), a linear function is assumed. It means that the number of trees decreases because the beetles feed on trees. So a novel tree-beetle model is obtained as

$$
\left\{\begin{array}{l}
\dot{V}(t)=r_{v} V(t)\left(1-\frac{V(t)}{k_{v}}\right)-\delta B(t) V(t) \\
\dot{B}(t)=r_{b} B(t)\left(1-\frac{B(t)}{k_{b}}\right)+\eta \delta B(t) V(t)-\frac{\alpha B^{2}(t)}{1+\beta B^{2}(t)}
\end{array}\right.
$$


where $\delta$ represents the rate that every beetle eats tree; $\eta$ is the conversion coefficient of the beetles; $\eta \delta B(t) V(t)$ denotes the number of the beetles increases after they eat trees; and $V(t)$ and $B(t)$ can be treated as the forest (tree) and pest (beetle). Thus, model (2) is called the forest-pest model. To a certain extent, they can also be seen as prey and predator.

Furthermore, we let $q_{1}$ represent the proportion of pests (prey) that is captured, transferred, or killed by using the cultural and chemical strategies. Therefore, the control model for $B(t)>$ ET is written as

$$
\left\{\begin{array}{c}
\dot{V}(t)=r_{v} V(t)\left(1-\frac{V(t)}{k_{v}}\right)-\delta B(t) V(t), \\
\dot{B}(t)=r_{b} B(t)\left(1-\frac{B(t)}{k_{b}}\right)+\eta \delta B(t) V(t) \\
\quad \frac{\alpha B^{2}(t)}{1+\beta B^{2}(t)}-q_{1} B(t)
\end{array}\right.
$$

where the ET is a switching threshold, i.e., system (2) is satisfied if $B(t)<$ ET while system (3) is satisfied if $B(t)>$ ET.

In the next subsection, some preliminaries of the Filippov system are provided to understand this paper easily, which prepares for the later sections.

2.2. Preliminaries. On the basis of IPM strategies and TPC, models (2) and (3) are combined and rewritten as $[11,51]$

$$
\left\{\begin{array}{c}
\dot{V}(t)=r_{v} V(t)\left(1-\frac{V(t)}{k_{v}}\right)-\delta B(t) V(t), \\
\dot{B}(t)=r_{b} B(t)\left(1-\frac{B(t)}{k_{b}}\right)+\eta \delta B(t) V(t) \\
-\frac{\alpha B^{2}(t)}{1+\beta B^{2}(t)}-\varepsilon q_{1} B(t)
\end{array}\right.
$$

with

$$
\varepsilon= \begin{cases}0, & B(t)<\mathrm{ET}, \\ 1, & B(t)>\mathrm{ET} .\end{cases}
$$

Models (4) and (5) are related to TPC. More details on the Filippov system have been given in the literature $[52,53]$. and

Let $H(Z)=B(t)-$ ET with column vector $Z=(V, B)^{T}$

$$
\begin{aligned}
F_{S_{1}}(Z)= & \left(r_{v} V\left(1-\frac{V}{k_{v}}\right)-\delta B V, r_{b} B\left(1-\frac{B}{k_{b}}\right)\right. \\
& \left.+\eta \delta B V-\frac{\alpha B^{2}}{1+\beta B^{2}}\right)^{T}, \\
F_{S_{2}}(Z)= & \left(r_{v} V\left(1-\frac{V}{k_{v}}\right)-\delta B V, r_{b} B\left(1-\frac{B}{k_{b}}\right)\right. \\
& \left.+\eta \delta B V-\frac{\alpha B^{2}}{1+\beta B^{2}}-q_{1} B\right)^{T} .
\end{aligned}
$$

Then, system (6) is rewritten as a Filippov system $[52,53]$ :

$$
\dot{Z}(t)= \begin{cases}F_{S_{1}}(Z), & Z \in S_{1}, \\ F_{S_{2}}(Z), & Z \in S_{2} .\end{cases}
$$

Moreover, the discontinuity boundary set is defined; that is, $\Sigma=\left\{Z \in R_{+}^{2} \mid H(Z)=0\right\}$, which divides $R_{+}^{2}$ into two regions, i.e.,

$$
\begin{aligned}
& S_{1}=\left\{Z \in R_{+}^{2} \mid H(Z)<0\right\}, \\
& S_{2}=\left\{Z \in R_{+}^{2} \mid H(Z)>0\right\} .
\end{aligned}
$$

In this paper, Filippov system (7) in region $S_{1}$ or $S_{2}$ is, respectively, referred to as subsystem $S_{1}$ or subsystem $S_{2}$.

Let

$$
\sigma(Z)=\left\langle H_{Z}(Z), F_{S_{1}}(Z)\right\rangle\left\langle H_{Z}(Z), F_{S_{2}}(Z)\right\rangle
$$

where $H_{Z}$ is a nonvanishing gradient of the smooth scale function on $\Sigma$ and $\langle\cdot\rangle$ denotes the standard scalar product. Then, sliding regions are defined as

$$
\Sigma_{S}=\{Z \in \Sigma \mid \sigma(Z) \leq 0\} .
$$

Meanwhile, the following regions on $\Sigma$ are distinguished:

(i) Escaping region: if $\left\langle H_{Z}(Z), F_{S_{1}}(Z)\right\rangle<0$ and $\left\langle H_{Z}(Z), F_{S_{2}}(Z)\right\rangle>0$

(ii) Sliding region: if $\left\langle H_{Z}(Z), F_{S_{1}}(Z)\right\rangle>0$ and $\left\langle H_{Z}(Z), F_{S_{2}}(Z)\right\rangle<0$

(iii) Sewing region: if $\left\langle H_{Z}(Z), F_{S_{1}}(Z)\right\rangle\left\langle H_{Z}(Z)\right.$, $\left.F_{S_{2}}(Z)\right\rangle>0$

The essential definitions for different types of equilibria of Filippov system (7) are taken from the literature [54, 55].

Definition 1. $Z^{*}$ is called a regular equilibrium of Filippov system (7) if $F_{S_{1}}\left(Z^{*}\right)=0$ and $H\left(Z^{*}\right)<0$, or $F_{S_{2}}\left(Z^{*}\right)=0$ and $H\left(Z^{*}\right)>0$. $Z^{*}$ is called a virtual equilibrium of Filippov system $(7)$ if $F_{S_{1}}\left(Z^{*}\right)=0$ and $H\left(Z^{*}\right)>0$, or $F_{S_{2}}\left(Z^{*}\right)=0$ and $H\left(Z^{*}\right)<0$.

Definition 2. $Z^{*}$ is called a pseudoequilibrium if it is an equilibrium on the sliding segment of Filippov system (7), i.e., $(1-\lambda(Z)) F_{S_{1}}\left(Z^{*}\right)+\lambda(Z) F_{S_{2}}\left(Z^{*}\right)=0, H\left(Z^{*}\right)=0$, and $0<\lambda(Z)<1$, where

$$
\lambda(Z)=\frac{\left\langle H_{Z}(Z), F_{S_{1}}(Z)\right\rangle}{\left\langle H_{Z}(Z), F_{S_{1}}(Z)-F_{S_{2}}(Z)\right\rangle} .
$$

Definition 3. $Z^{*}$ is called a boundary equilibrium of Filippov system (7) if $F_{S_{1}}\left(Z^{*}\right)=0$ with $H\left(Z^{*}\right)=0$ or $F_{S_{2}}\left(Z^{*}\right)=0$ with $H\left(Z^{*}\right)=0$.

Definition 4. $Z^{*}$ is called a tangent point of Filippov system (7) if $Z^{*} \in \Sigma_{S}$ and $\left\langle H_{Z}\left(Z^{*}\right), F_{S_{1}}\left(Z^{*}\right)\right\rangle=0$ or $\left\langle H_{Z}\left(Z^{*}\right)\right.$, $\left.F_{S_{2}}\left(Z^{*}\right)\right\rangle=0$. 


\section{Equilibria and Sliding Dynamics}

This section discusses conditions for the existence and stability of equilibria of subsystems $S_{1}$ and $S_{2}$. The sliding segment/region and equilibria of Filippov system (7) are addressed, which prepares for Section 4.

3.1. Existence of Equilibria for Subsystems $S_{1}$ and $S_{2}$. To discuss the types of equilibria and sliding bifurcations of Filippov system (7), the existence of equilibria for subsystems $S_{1}$ and $S_{2}$ needs to be solved. For subsystem $S_{1}$, let $\dot{V}(t)=0$ and $\dot{B}(t)=0$. Then, the equilibrium $E_{1 *}\left(V_{1 *}, B_{1 *}\right)$ satisfies

$$
B^{3}(t)+a_{1} B^{2}(t)+b_{1} B(t)+c_{1}=0,
$$

where $a_{1}=\left(\left(-k_{b} r_{b} r_{v}-\eta \delta k_{b} k_{v} r_{v}\right) /\left(r_{b} r_{v}+\delta^{2} \eta k_{b} k_{v}\right)\right), \quad b_{1}=$ $\left(\left(r_{b} r_{v}+\delta^{2} \eta k_{b} k_{v}+\alpha k_{b} r_{v}\right) /\left(r_{b} r_{v} \beta+\delta^{2} \eta k_{b} k_{v} \beta\right)\right)$, and $c_{1}=$ $\left(\left(-k_{b} r_{b} r_{v}-\eta \delta k_{b} k_{v} r_{v}\right) /\left(r_{b} r_{v} \beta+\delta^{2} \eta k_{b} k_{v} \beta\right)\right)$. Let $Q_{1}=\left(\left(a_{1}^{2}-\right.\right.$ $\left.\left.3 b_{1}\right) / 9\right), R_{1}=-\left(\left(2 a_{1}^{3}-9 a_{1} b_{1}+27 c_{1}\right) / 54\right), M_{1}=R_{1}^{2}-Q_{1}^{3}$, and $\theta_{1}=\arccos \left(R_{1} / Q_{1}^{3 / 2}\right)$.

(i) If $M_{1}<0$, then (12) has three fixed solutions given by

$$
\begin{cases}B_{1 i}=-2 \sqrt{Q_{1}} \cos \left(\frac{\theta_{1}+2 k \pi}{3}\right)-\frac{a_{1}}{3}, & k=-1,0,1 ; \\ V_{1 i}=k_{v}-\frac{\delta B_{1 i} k_{v}}{r_{v}} & i=1,2,3,\end{cases}
$$

(ii) If $M_{1}>0$, then (12) has a unique equilibrium point given by

$$
\left\{\begin{array}{l}
B_{1}=\left(-R_{1}+\sqrt{M_{1}}\right)^{1 / 3}+\left(-R_{1}-\sqrt{M_{1}}\right)^{1 / 3}-\frac{a_{1}}{3} \\
V_{1}=k_{v}-\frac{\delta B_{1} k_{v}}{r_{v}}
\end{array}\right.
$$

(iii) If $M_{1}=0$, then (12) has two equilibrium points. We do not discuss this case in the present paper.

In particular, the equilibrium $E_{1}^{*}$ must be positive, i.e., $V_{1 i}>0, B_{1 i}>0(i=1,2,3)$. Analogously, the existence conditions of equilibria for subsystem $S_{2}$ can be obtained.

3.2. Stability of Equilibria for Subsystems $S_{1}$ and $S_{2}$. In general, the Jacobian matrix is employed to explore the stability of equilibrium $E_{1 *}\left(V_{1 *}, B_{1 *}\right)$ for subsystem $S_{1}$, i.e.,

$$
J_{1 *}=\left(\begin{array}{cc}
r_{v}-\frac{2 r_{v} V_{1 *}}{k_{v}}-\delta B_{1 *} & -\delta V_{1 *} \\
\eta \delta B_{1 *} & r_{b}-\frac{2 r_{b} B_{1 *}}{k_{b}}+\eta \delta V_{1 *}-\frac{2 \alpha B_{1 *}}{\left(1+\beta B_{1 *}^{2}\right)^{2}}
\end{array}\right) .
$$

Then, the characteristic equation is $\lambda^{2}+p_{1} \lambda+q_{1}=0$, where

$$
p_{1}=-r_{v}+\frac{2 r_{v} V_{1 *}}{k_{v}}+\delta B_{1 *}-r_{b}+\frac{2 r_{b} B_{1 *}}{k_{b}}-\eta \delta V_{1 *}+\frac{2 \alpha B_{1 *}}{\left(1+\beta B_{1 *}^{2}\right)^{2}},
$$

$$
\begin{aligned}
q_{1}= & \left(r_{v}-\frac{2 r_{v} V_{1 *}}{k_{v}}-\delta B_{1 *}\right)\left(r_{b}-\frac{2 r_{b} B_{1 *}}{k_{b}}+\eta \delta V_{1 *}\right. \\
& \left.-\frac{2 \alpha B_{1 *}}{\left(1+\beta B_{1 *}^{2}\right)^{2}}\right)+\eta \delta^{2} B_{1 *} V_{1 *}
\end{aligned}
$$

If inequalities $p_{1}>0$ and $q_{1}>0$ are satisfied, then $E_{1 *}$ is asymptotically stable. Similarly, the conditions for the stability of equilibria of subsystem $S_{2}$ are addressed.

3.3. Sliding Segment and Region. From Definition 2, the equation is obtained:

$$
\lambda(Z)=\frac{r_{b}\left(1-\left(B(t) / k_{b}\right)\right)+\eta \delta V(t)-\left(\alpha B(t) /\left(1+\beta B^{2}(t)\right)\right)}{q_{1}} .
$$

The sliding regions are determined by solving $0 \leq \lambda(Z) \leq 1$ with respect to $V(t)$. Thus, the algebraic equations need to be considered, i.e.,

$$
\left\{\begin{array}{l}
r_{b}\left(1-\frac{\mathrm{ET}}{k_{b}}\right)+\eta \delta V(t)-\frac{\alpha \mathrm{ET}}{1+\beta \mathrm{ET}^{2}}=0 \\
r_{b}\left(1-\frac{\mathrm{ET}}{k_{b}}\right)+\eta \delta V(t)-\frac{\alpha \mathrm{ET}}{1+\beta \mathrm{ET}^{2}}-q_{1}=0
\end{array}\right.
$$

Then, solving equation (19) with respect to $V(t)$ yields two real roots:

$$
\begin{aligned}
& V_{1}=\frac{\left(\alpha \mathrm{ET} /\left(1+\beta \mathrm{ET}^{2}\right)\right)-r_{b}\left(1-\left(\mathrm{ET} / k_{b}\right)\right)}{\eta \delta}, \\
& V_{2}=\frac{q_{1}+\left(\alpha \mathrm{ET} /\left(1+\beta \mathrm{ET}^{2}\right)\right)-r_{b}\left(1-\left(\mathrm{ET} / k_{b}\right)\right)}{\eta \delta} .
\end{aligned}
$$

Because of the relation between $V_{1}$ and $V_{2}\left(V_{1}<V_{2}\right)$, the sliding segment of Filippov system (7) can be defined as

$$
\Sigma_{S}=\left\{(V, B) \mid V_{1} \leq V \leq V_{2}, B=\mathrm{ET}\right\} .
$$

3.4. Equilibria of the Filippov System. Nullclines of both subsystems $S_{1}$ and $S_{2}$ are related to the existence of equilibria, which is conducive to sliding bifurcation analysis and to estimating the existence of equilibria of Filippov system (7). For the two subsystems, nullclines are determined by using equations $\dot{V}(t)=0$ and $\dot{B}(t)=0$ as 


$$
\begin{aligned}
& f_{S_{1}}=f_{S_{2}}=\frac{k_{v}}{r_{v}}\left(r_{v}-\delta B(t)\right), \\
& g_{S_{1}}=\frac{1}{\eta \delta}\left(-r_{b}\left(1-\frac{B(t)}{k_{b}}\right)+\frac{\alpha B(t)}{1+\beta B^{2}(t)}\right), \\
& g_{S_{2}}=\frac{1}{\eta \delta}\left(q_{1}-r_{b}\left(1-\frac{B(t)}{k_{b}}\right)+\frac{\alpha B(t)}{1+\beta B^{2}(t)}\right) .
\end{aligned}
$$

Different types of equilibria of Filippov system (7) were defined in Section 2. Several types of equilibria for Filippov system (7) are defined, which include the regular equilibrium, virtual equilibrium, pseudoequilibrium, boundary equilibrium, and a special point called the tangent point. These equilibria are denoted by $E^{R}, E^{V}$, $E^{P}, E^{B}$, and $E^{T}$. For Filippov system (7), more detailed definitions of the equilibria mentioned are in the following section.

3.4.1. Regular/Virtual Equilibrium. According to Definition $1, Z_{j i}=\left(V_{j i}, B_{j i}\right)(j=1,2 ; i=1,2,3)$ is a regular equilibrium of Filippov system (7) if $F_{S_{j}}\left(Z_{j i}\right)=0$, for subsystem $S_{1}$ (i.e., $j=1$ ), $B_{1 i}<$ ET, or for subsystem $S_{2}$ (i.e., $j=2$ ), $B_{2 i}>$ ET. These equilibria are denoted by $E_{1 i}^{R}$ and $E_{2 i}^{R}$, respectively. $Z_{j i}=\left(V_{j i}, B_{j i}\right)(j=1,2 ; i=1,2,3)$ is a virtual equilibrium of Filippov system (7) if $F_{S_{i}}\left(Z_{j i}\right)=0$, for subsystem $S_{1}$ (i.e., $j=1$ ), $B_{1 i}>\mathrm{ET}$, or for subsystem $S_{2}$ (i.e., $j=2$ ), $B_{2 i}<\mathrm{ET}$. These equilibria are denoted by $E_{1 i}^{V}$ and $E_{2 i}^{V}$, respectively.

3.4.2. Pseudoequilibrium. According to Definition 2, by employing the Utkin equivalent control method [51] and letting $H_{Z}=\dot{B}(t)=0$, equations with respect to $\varepsilon$ are obtained:

$$
\varepsilon=\frac{r_{b}\left(1-\left(\mathrm{ET} / k_{b}\right)\right)+\eta \delta V-\left(\alpha \mathrm{ET} /\left(1+\beta \mathrm{ET}^{2}\right)\right)}{q_{1}} .
$$

Thus, the equation $\dot{V}(t)=r_{v} V(t)\left(1-\left(V(t) / k_{v}\right)\right)-$ $\delta B(t) V(t)$ determines the dynamics on $\Sigma_{S}$. If pseudoequilibrium $E^{P}\left(V_{P}\right.$, ET) satisfies $\dot{V}(t)=0$ (i.e., $V_{P}=\left(\left(k_{v}\left(r_{v}-\right.\right.\right.$ $\left.\delta B(t))) / r_{v}\right)$ and $\left.B(t)=\mathrm{ET}\right)$, then $E^{P}\left(\left(k_{v}\left(r_{v}-\delta \mathrm{ET}\right) / r_{v}\right)\right.$, ET) $\in \Sigma_{S}$. Therefore, $V_{P}$ must satisfy the inequality $V_{1} \leq V_{P} \leq V_{2}$, i.e., if the inequalities

$$
\begin{aligned}
& \frac{k_{b} k_{v} \delta^{2} \eta \mathrm{ET}}{k_{b} k_{v} \delta \eta+r_{b}\left(k_{b}-\mathrm{ET}\right)-\left(\alpha \mathrm{ET} k_{b} /\left(1+\beta \mathrm{ET}^{2}\right)\right)} \\
& <r_{v}<\frac{k_{b} k_{v} \delta^{2} \eta \mathrm{ET}}{k_{b} k_{v} \delta \eta-q_{1}+r_{b}\left(k_{b}-\mathrm{ET}\right)-\left(\alpha \mathrm{ET}_{b} / 1+\beta \mathrm{ET}^{2}\right)}
\end{aligned}
$$

hold, then Filippov system (7) has a pseudoequilibrium $E^{P}$.

3.4.3. Boundary Equilibrium. According to Definition 3, the boundary equilibrium of Filippov system (7) satisfies

$$
\left\{\begin{array}{l}
r_{v} V(t)\left(1-\frac{V(t)}{k_{v}}\right)-\delta B(t) V(t)=0, \\
r_{b}\left(1-\frac{B(t)}{k_{b}}\right)+\eta \delta V(t)-\frac{\alpha B(t)}{1+\beta B^{2}(t)}-q_{1}=0, \\
B(t)=\mathrm{ET}
\end{array}\right.
$$

with $\varepsilon=0$ or 1 . If $\varepsilon=0$, the boundary equilibrium

$$
E_{1}^{B}=\left(\frac{\left(\alpha \mathrm{ET} /\left(1+\beta \mathrm{ET}^{2}\right)\right)-r_{b}\left(1-\left(\mathrm{ET} / k_{b}\right)\right)}{\eta \delta}, \mathrm{ET}\right),
$$

is obtained. If $\varepsilon=1$, we have the boundary equilibrium

$$
E_{2}^{B}=\left(\frac{q_{1}+\left(\alpha \mathrm{ET} /\left(1+\beta \mathrm{ET}^{2}\right)\right)-r_{b}\left(1-\left(\mathrm{ET} / k_{b}\right)\right)}{\eta \delta}, \mathrm{ET}\right) .
$$

3.4.4. Tangent Point. According to Definition 4, the tangent point $E^{T}\left(V_{T}, \mathrm{ET}\right)$ on $\Sigma_{S}$ satisfies

$$
\left\{\begin{array}{l}
r_{b}\left(1-\frac{B(t)}{k_{b}}\right)+\eta \delta V(t)-\frac{\alpha B(t)}{1+\beta B^{2}(t)}-q_{1}=0, \\
B(t)=\mathrm{ET} .
\end{array}\right.
$$
yields

Solving equations (28) with respect to $V(t)$ and $B(t)$

$$
\begin{aligned}
& E_{1}^{T}=\left(\frac{\left(\alpha \mathrm{ET} /\left(1+\beta \mathrm{ET}^{2}\right)\right)-r_{b}\left(1-\left(\mathrm{ET} / k_{b}\right)\right)}{\eta \delta}, \mathrm{ET}\right), \\
& E_{2}^{T}=\left(\frac{q_{1}+\left(\alpha \mathrm{ET} /\left(1+\beta \mathrm{ET}^{2}\right)\right)-r_{b}\left(1-\left(\mathrm{ET} / k_{b}\right)\right)}{\eta \delta}, \mathrm{ET}\right) .
\end{aligned}
$$

\section{Numerical Simulation}

In this section, four cases of sliding bifurcations for Filippov system (7) with different equilibria of subsystems $S_{1}$ and $S_{2}$ are investigated, which are summarized in Table 1.

4.1. Case $A$. Subsystem $S_{1}$ has an unstable equilibrium point, and there exist stable limit cycles. There are three cases of subsystem $S_{2}$ :

(i) Subsystem $S_{2}$ has only an unstable equilibrium point and stable limit cycles

(ii) Subsystem $S_{2}$ is a bistable state

(iii) Subsystem $S_{2}$ has only one stable equilibrium point

Figure 1(a) shows that there is only one unstable equilibrium $E_{11}$ and stable limit cycles in subsystem $S_{1}$. Figure 1(b) shows that subsystem $S_{2}$ is similar to subsystem $S_{1}$ in that there is only one unstable equilibrium $E_{21}$ and stable limit cycles. Figure 1(c) shows that there are three 
TABLE 1: The summary of numerical simulation.

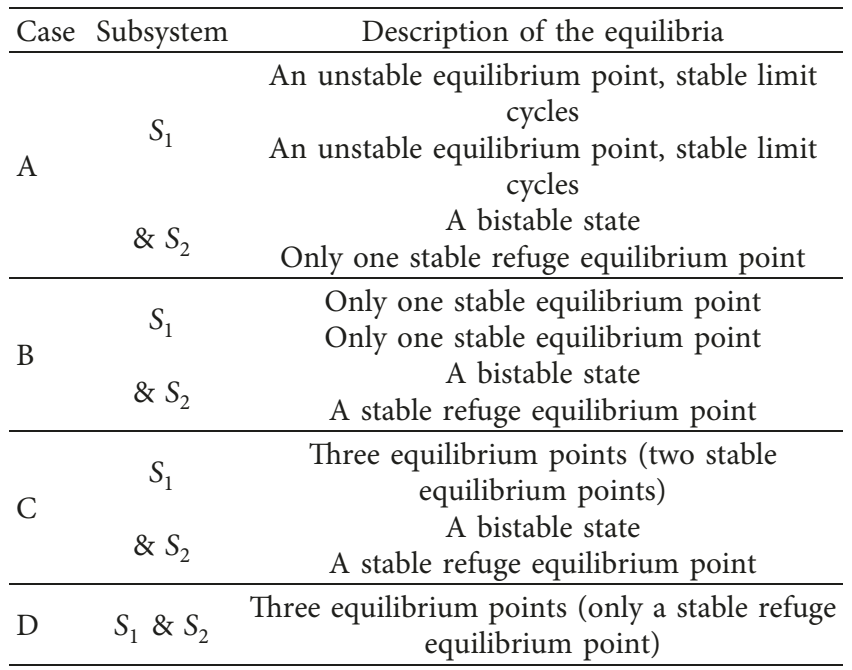

equilibria (i.e., $E_{21}, E_{22}$ and $E_{23}$ ) in subsystem $S_{2} . E_{21}$ and $E_{23}$ are stable, i.e., subsystem $S_{2}$ is a bistable state. Figure $1(\mathrm{~d})$ shows that there is only one stable equilibrium $E_{23}$, a stable refuge equilibrium point, in subsystem $S_{2}$.

The sliding mode phenomena in the three subcases of Case A are discussed as follows.

Case A.1: subsystem $S_{2}$ has only one unstable equilibrium point and stable limit cycles.

Figure 2(a) shows the sliding bifurcation of the Filippov system when the value of ET varies in the range [3.8, 5.8]. $V_{1}$ and $V_{2}$ are two endpoints of the sliding segment. Moreover, sliding limit cycles appear. Figures 2(b)-2(d) present the three cases of Figure 2(a). In Figure 2(b), if $B_{11}>B_{21}>\mathrm{ET}(\mathrm{ET}=3.9)$, Filippov system (7) has limit cycles and stabilizes to the larger limit cycle and virtual equilibria $E_{11}^{V}$ of subsystem $S_{1}$. In Figure 2(c), if $B_{11}>\mathrm{ET}>B_{21}(\mathrm{ET}=4.8)$, the Filippov system has a pseudoequilibrium $E^{P}$ and stabilizes on the sliding segment with $E^{P}$. In Figure 2(d), if $\mathrm{ET}>B_{11}>B_{21}(\mathrm{ET}=5.9)$, there are limit cycles. Filippov system (7) stabilizes to the larger limit cycle and virtual equilibria $E_{21}^{V}$ of subsystem $S_{2}$.

The results show that the Filippov system has limit cycles regardless of the initial values $(V(t)$ and $B(t))$ and stabilizes to the larger limit cycle. In other words, when $q_{1}=0.2$, the pest population density stabilizes in a relatively small range, i.e., no pest outbreaks will occur. Other interesting problems can be considered, for example, how to decide the time and choose strategies for controlling pests, how to determine the equilibria of the Filippov system, and how to find regions of pseudoequilibrium when there is sliding bifurcation. To solve these problems, sliding bifurcation diagrams with nullclines of the Filippov system for different situations are investigated.

Case A.2: subsystem $S_{2}$ is a bistable state.

In Figure 3, $V_{1} V_{2}$ is the sliding segment. In this section, for the Filippov system, red, black and magenta dashed lines, respectively, indicate nullclines $f_{S_{1}} / f_{S_{2}}, g_{S_{1}}$, and $g_{S_{2}}$. The points of intersection between the nullclines are equilibria of the Filippov system. In Figure 3(a), when the ET is set as different values in the range $[2,9]$, the Filippov system shows sliding bifurcation and limit cycles. The difference compared with Figure 2(a) is that there is only one larger limit cycle. Subsystem $S_{1}$ has the equilibrium $E_{11}$ while subsystem $S_{2}$ has the three equilibria $E_{21}, E_{22}$, and $E_{23}$. In particular, $E_{21}$ and $E_{23}$ are stable. Therefore, subsystem $S_{2}$ is a bistable state. Figures 3(b)-3(d) present the three cases of Figure 3(a), i.e., if $B_{11}>B_{21}>\mathrm{ET}>B_{22}>B_{23}(\mathrm{ET}=3)$, regardless of the initial values, the Filippov system is stable at $E_{21}$. $E_{21}$ is regular and denoted by $E_{21}^{R}$ (see Figure $3(\mathrm{~b})$ ). If $B_{11}>\mathrm{ET}>B_{21}>B_{22}>B_{23} \quad(\mathrm{ET}=4.7)$, the Filippov system has a pseudoequilibrium $E^{P}$ but no sliding limit cycle, and it stabilizes on the $V_{1} V_{2}$ with $E^{P}$ (see Figure 3(c)). If $\mathrm{ET}>B_{11}>B_{21}>B_{22}>B_{23}(\mathrm{ET}=6.45)$, the Filippov system has only one sliding limit cycle and stabilizes to this limit cycle (see Figure 3(d)). Therefore, when $q_{1}=0.45$, the pest population density will be stable in a larger range than in the case of $q_{1}=0.2$ as shown in Figure 1.

We conclude that the equilibria $\left(E_{11}, E_{21}, E_{22}\right.$, and $\left.E_{23}\right)$ are the points of intersection of nullclines (i.e., $f_{S_{1}} / f_{S_{2}}$, $g_{S_{1}}$, and $\left.g_{S_{2}}\right)$ for the Filippov system. The endpoints $\left(V_{1}\right.$ and $V_{2}$ ) of the sliding segment are located on two nullclines (i.e., $g_{S_{1}}$ and $g_{S_{2}}$.) The pseudoequilibrium is the point of intersection of the sliding segment and nullclines of the Filippov system. In addition, the pseudoequilibrium exists on the sliding segment $\left(E_{11} E_{21}\right)$. These findings are also confirmed in the following discussions.

Case A.3: subsystem $S_{2}$ has only one stable refuge equilibrium point.

Figure 4(a) presents the sliding bifurcation of the Filippov system for the different values of ET in the range $[0.5,7.8]$. Subsystem $S_{2}$ has only one stable equilibrium $E_{21}$. Figures $4(\mathrm{~b})-4(\mathrm{~d})$ present the three cases of Figure $4(\mathrm{a})$. If $B_{11}>\mathrm{ET}>B_{21}(\mathrm{ET}=1.54)$, the Filippov system has a pseudoequilibrium $E^{P}$ and stabilizes on the sliding segment $V_{1} V_{2}$ with $E^{P}$. There exists $E^{P}$ on the $E_{11} E_{21}$ (see Figure $\left.4(\mathrm{~b})\right)$. If $B_{11}>B_{21}>\mathrm{ET}(\mathrm{ET}=0.5)$, the ET is sufficiently small for the Filippov system to be stable at a refuge equilibrium point (see Figure $4(\mathrm{c})$ ). If $\mathrm{ET}>B_{11}>B_{21} \quad(\mathrm{ET}=6.76)$, regardless of the initial values, there is a sliding limit cycle and the Filippov system stabilizes to the limit cycle as shown in Figure $4(\mathrm{~d})$. Hence, when $q_{1}=0.6$, the pest population density will be stable in a smaller range than in the case of $q_{1}=0.45$ as shown in Figure 3 .

Therefore, when $q_{1}=0.45$, the range of pests that can be controlled is largest. At the same time, the strategy is the most suitable for controlling pests.

4.2. Case B. Subsystem $S_{1}$ has a stable equilibrium point while there are three cases of subsystem $S_{2}$. 


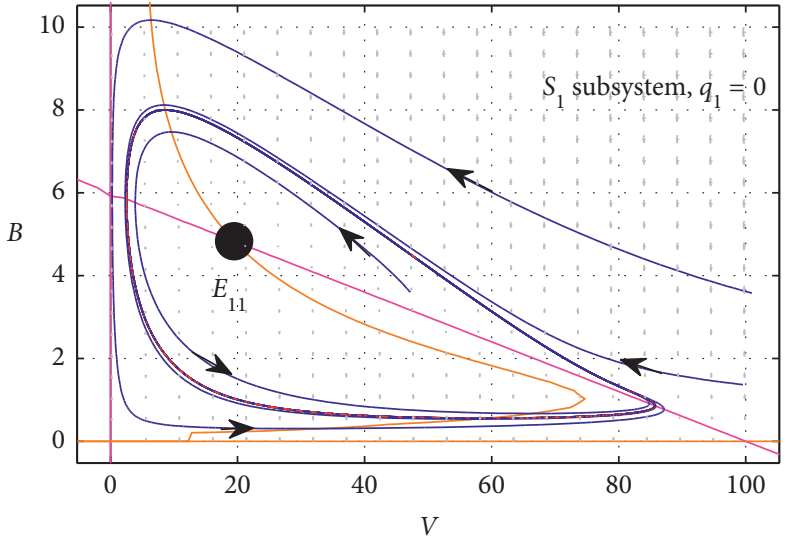

(a)

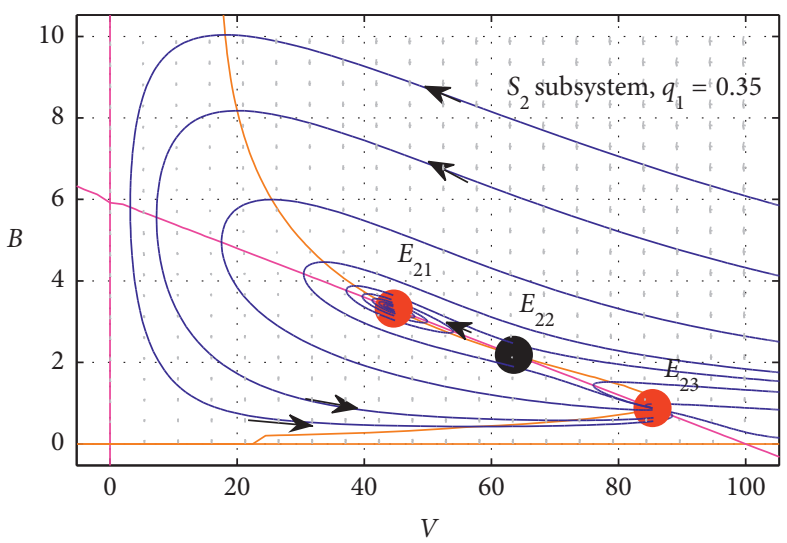

(c)

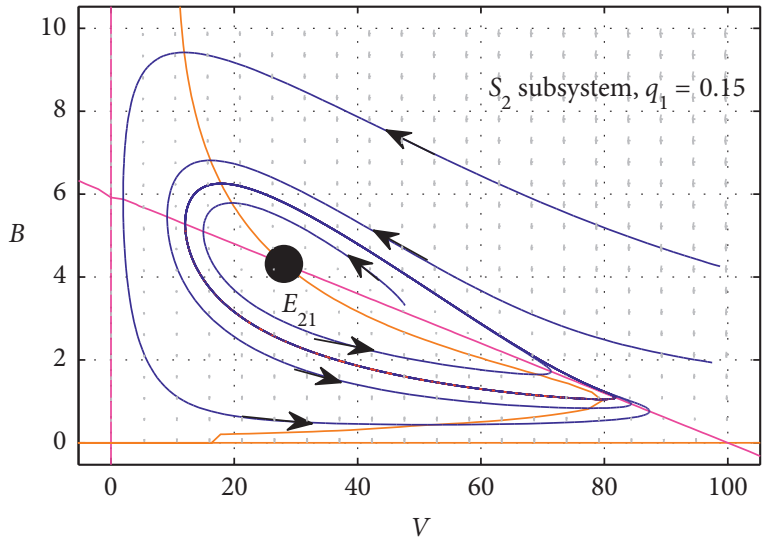

(b)

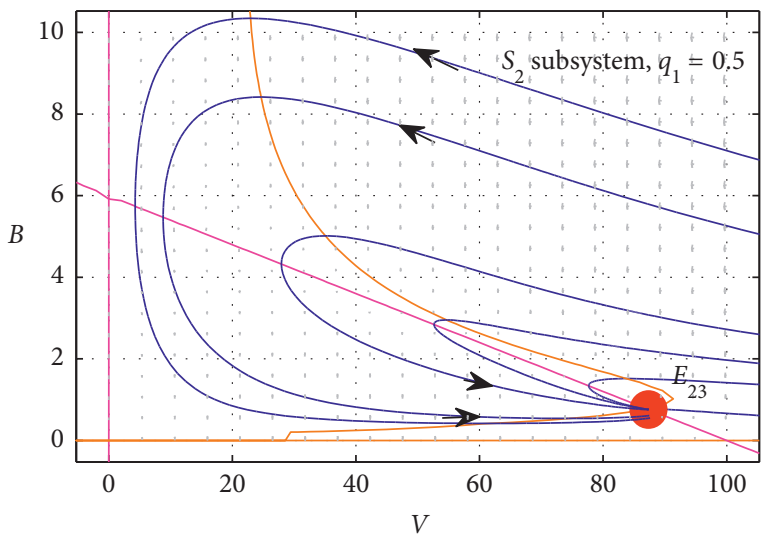

(d)

Figure 1: Existence of equilibria for Case A of Filippov system (7). Parameters are $r_{b}=1, k_{b}=20, \eta=0.06, \delta=0.5, \alpha=6, \beta=1, r_{v}=3$, and $k_{v}=100$. (a) $q_{1}=0$; (b) $q_{1}=0.15$; (c) $q_{1}=0.35$; (d) $q_{1}=0.5$.

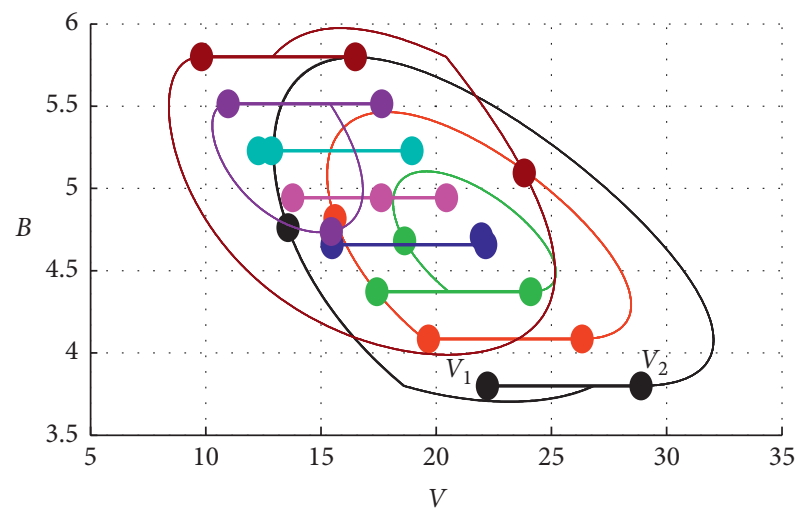

(a)

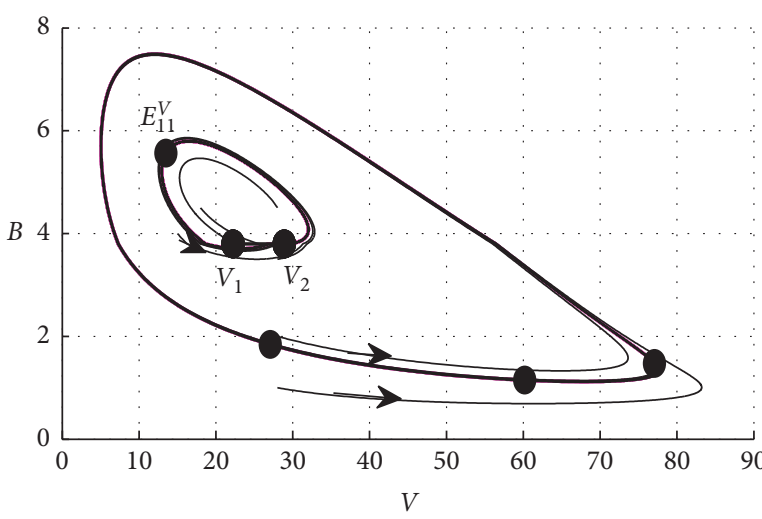

(b)

FIgUre 2: Continued. 


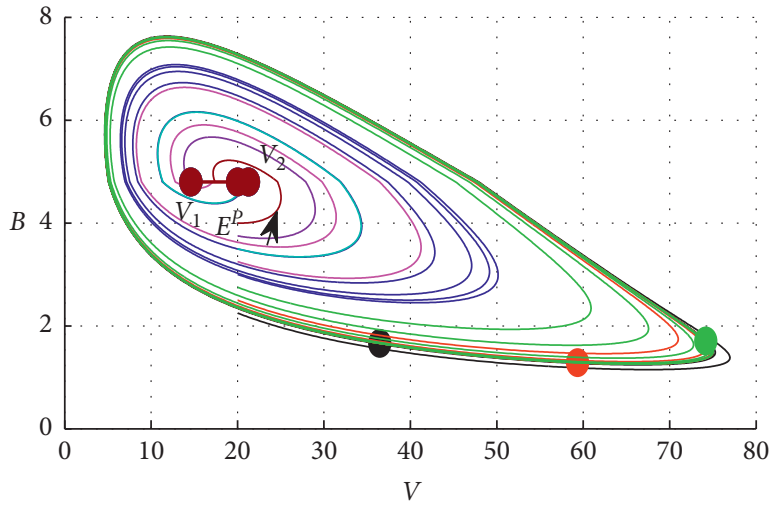

(c)

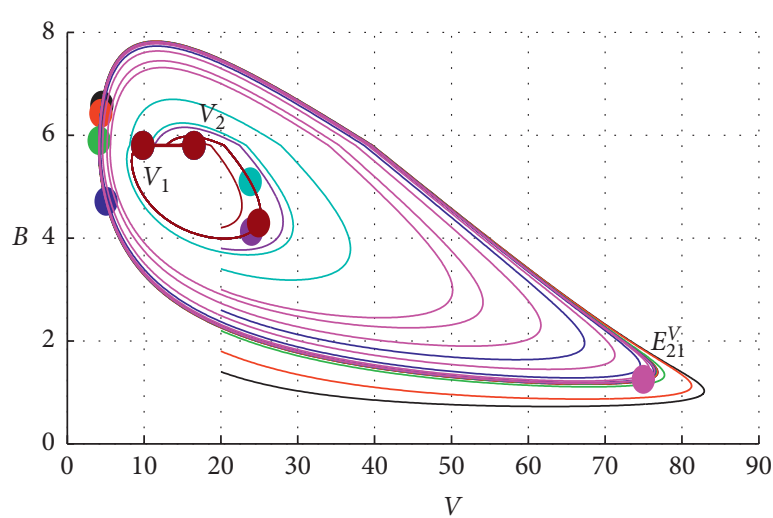

(d)

Figure 2: Sliding bifurcation for Case A.1 of Filippov system (7). Subsystem $S_{2}$ has only one unstable equilibrium point, and stable limit cycles exist. $q_{1}=0.2$, the other parameters are the same as those in Figure 1. (a) ET $\in[3.8,5.8]$; (b) ET $=3.9$; (c) ET $=4.8$; (d) ET $=5.9$.

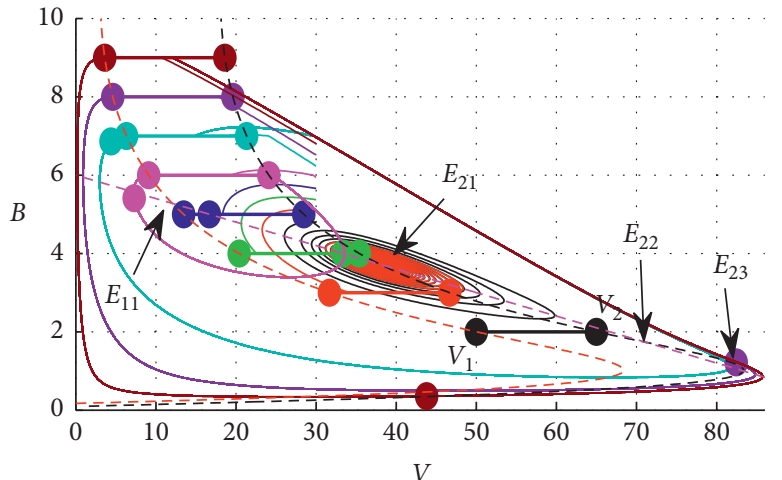

(a)

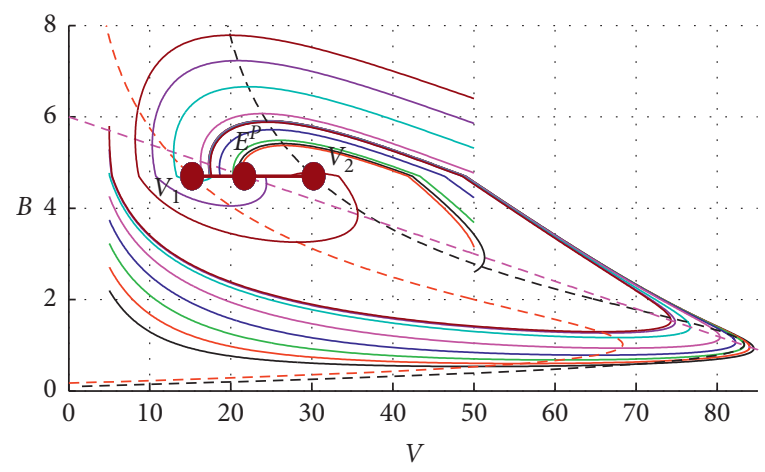

(c)

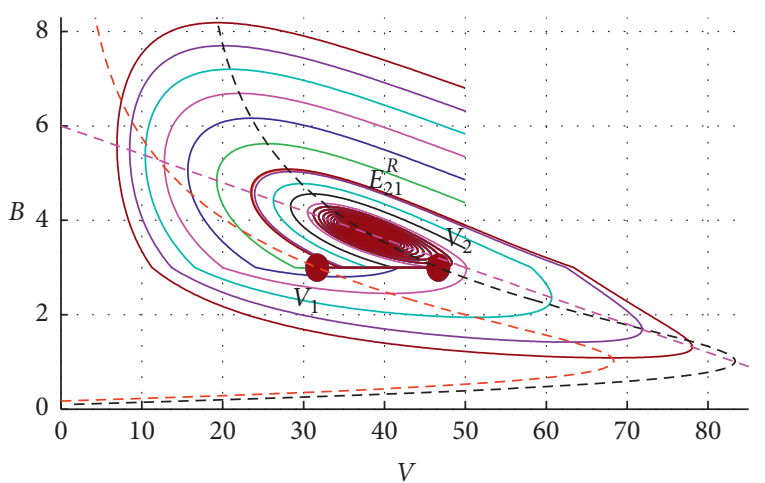

(b)

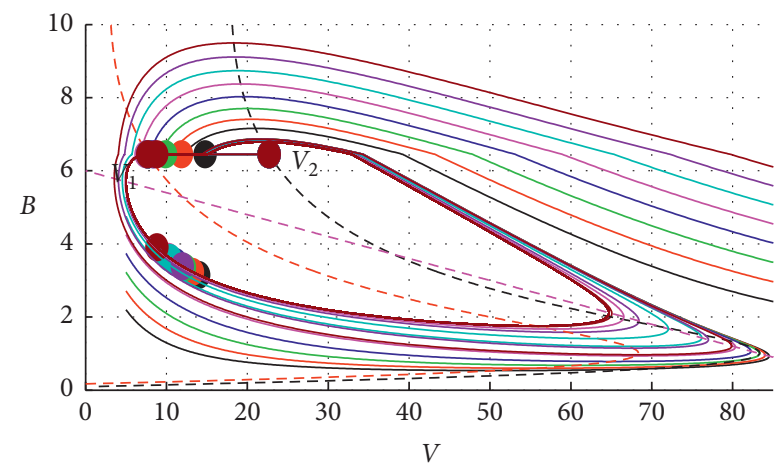

(d)

Figure 3: Sliding bifurcation for Case A.2 of Filippov system (7). Subsystem $S_{2}$ is a bistable state. $q_{1}=0.45$, the other parameters are the same as those in Figure 1. (a) ET $\in[2,9]$; (b) $\mathrm{ET}=3$; (c) $\mathrm{ET}=4.7$; (d) $\mathrm{ET}=6.45$.

(i) Subsystem $S_{2}$ has only one stable equilibrium point

(ii) Subsystem $S_{2}$ has two stable equilibrium points (i.e., Subsystem $S_{2}$ is a bistable state)

(iii) Subsystem $S_{2}$ has only one stable refuge equilibrium point

In Figure 5(a), only one stable equilibrium $E_{11}$ emerges in subsystem $S_{1}$. In Figure 5(b), subsystem $S_{2}$ has only one stable equilibrium $E_{21}$. In Figure 5(c), subsystem $S_{2}$ has two stable equilibria $E_{21}$ and $E_{23}$, which is a bistable state. In Figure 5(d), subsystem $S_{2}$ has a stable equilibrium $E_{23}$ that becomes a stable refuge equilibrium point with relatively small number of pests.

The sliding mode phenomena in the three subcases of Case $\mathrm{B}$ are discussed as follows:

Case B.1: subsystem $S_{2}$ has a stable equilibrium point. 


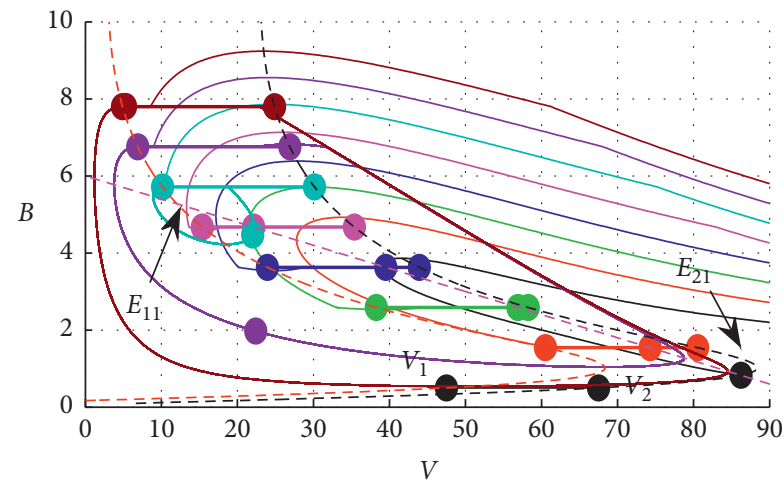

(a)

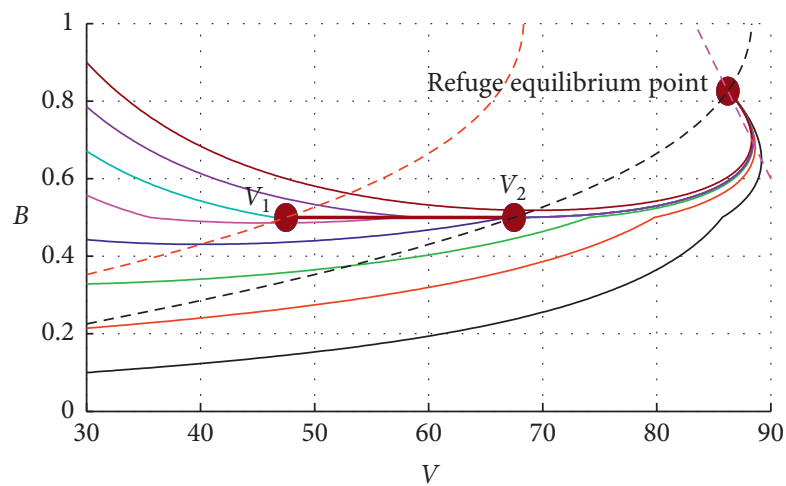

(c)

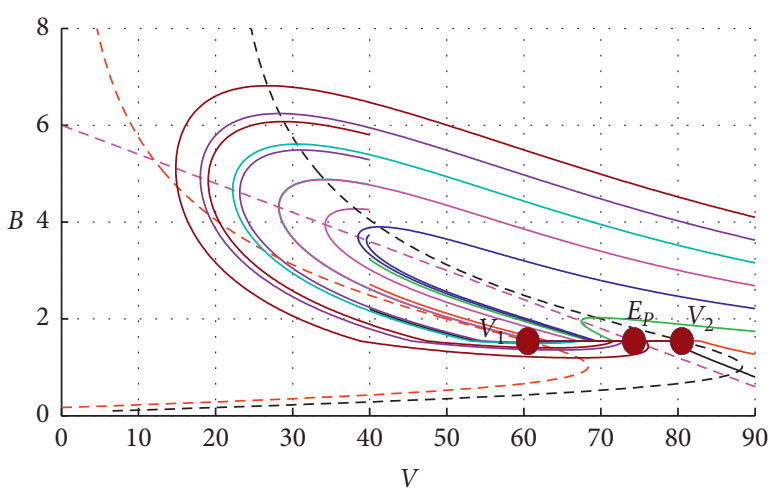

(b)

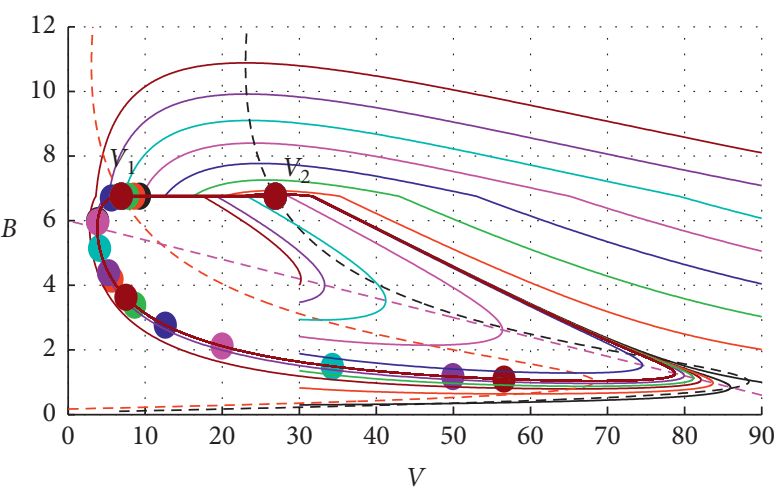

(d)

FIgURE 4: Sliding bifurcation for Case A.3 of Filippov system (7). Subsystem $S_{2}$ has only one stable refuge equilibrium point. $q_{1}=0.6$, the other parameters are the same as those in Figure 1. (a) ET $\in[0.5,7.8]$; (b) ET $=1.54$; (c) ET $=0.5$; (d) ET $=6.76$.

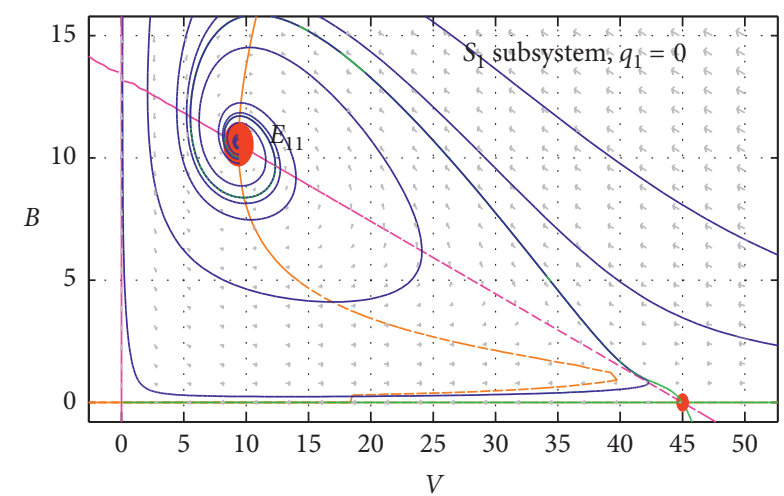

(a)

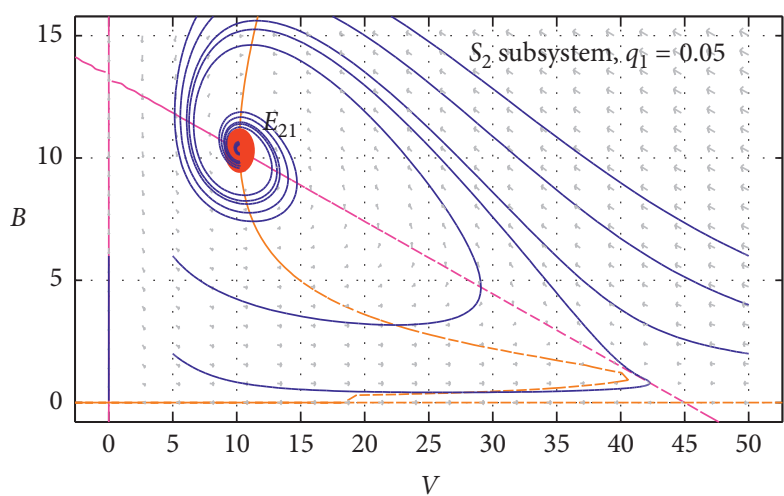

(b)

Figure 5: Continued. 


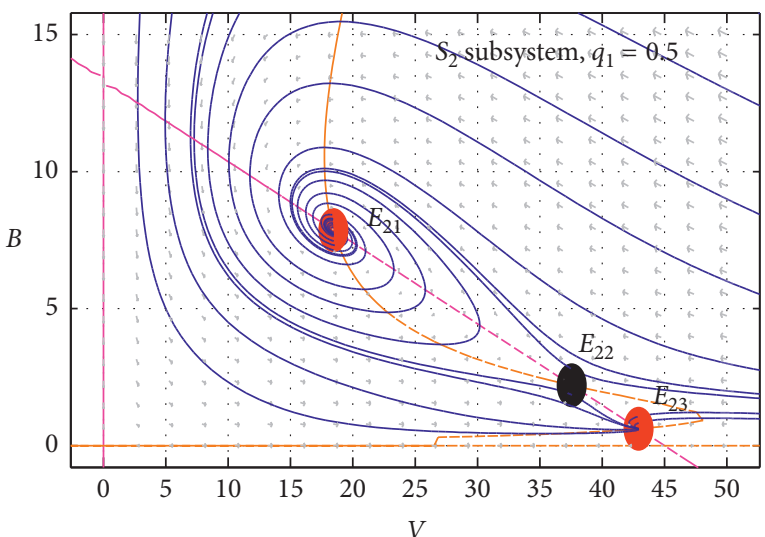

(c)

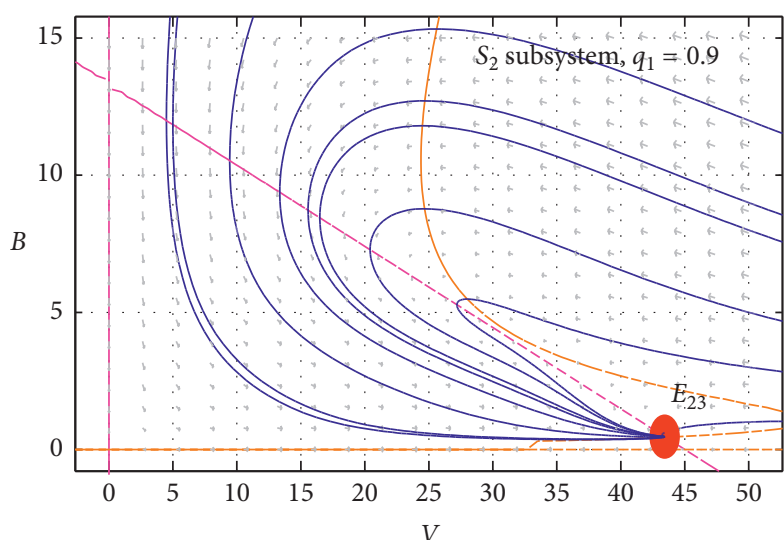

(d)

Figure 5: Existence of equilibria for Case B of Filippov system (7). Parameters are fixed as $r_{b}=0.5, k_{b}=10, \eta=0.4, \delta=0.15, \alpha=5.7, \beta=1$, $r_{v}=2$, and $k_{v}=45$. (a) $q_{1}=0$; (b) $q_{1}=0.05$; (c) $q_{1}=0.5$; (d) $q_{1}=0.9$.

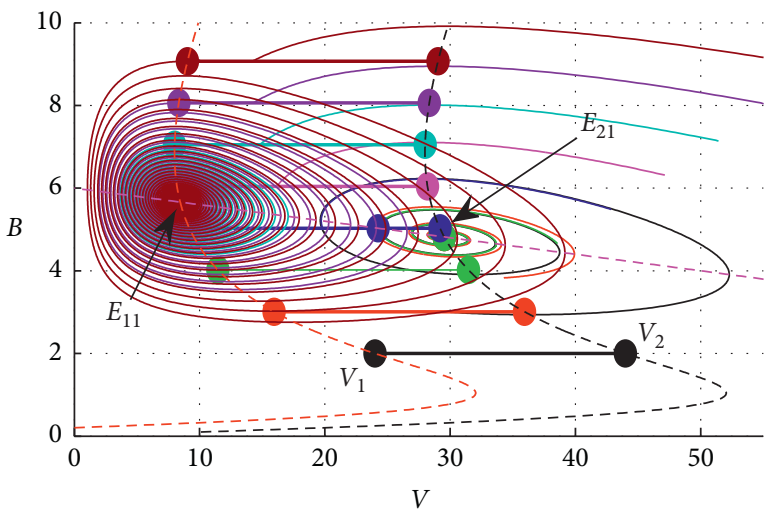

(a)

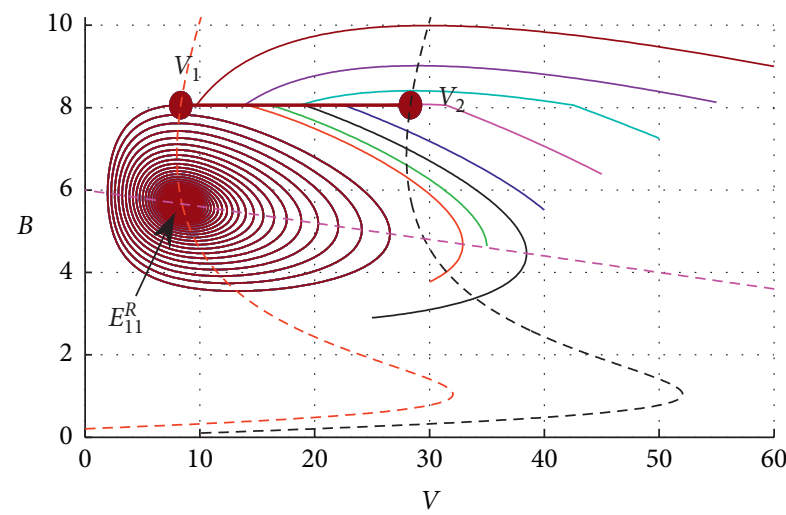

(c)

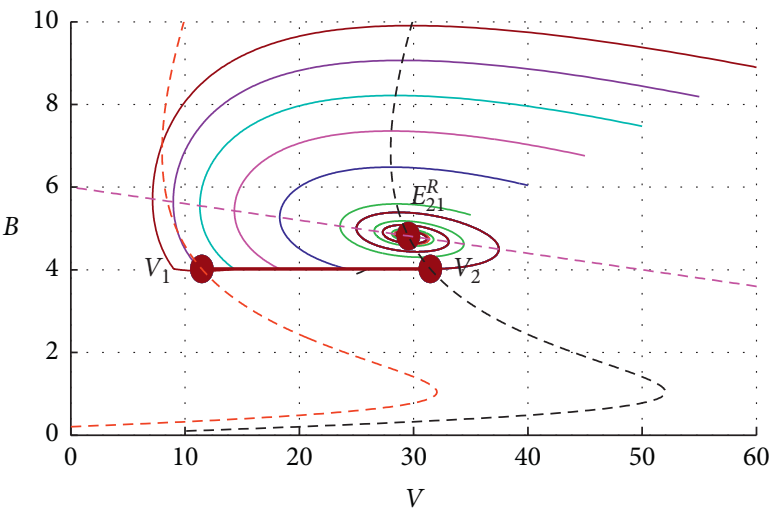

(b)

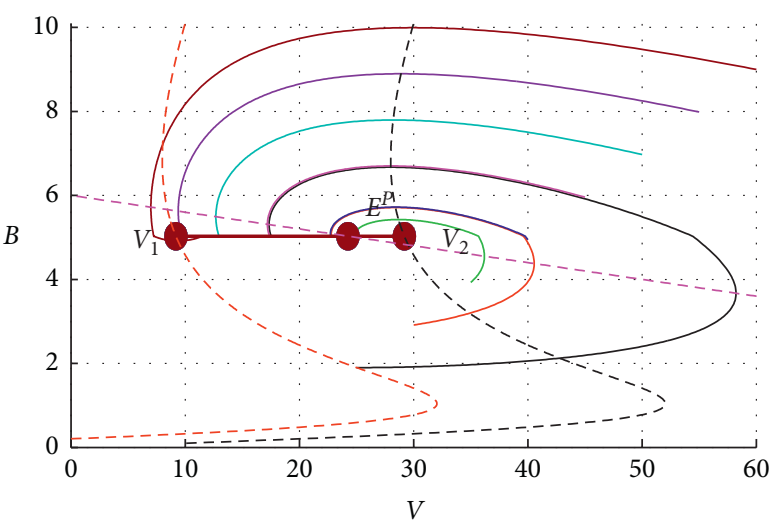

(d)

Figure 6: Sliding bifurcation for Case B.1 of Filippov system (7). Both subsystem $S_{1}$ and subsystem $S_{2}$ have one stable equilibrium point. $q_{1}=0.6$, the other parameters are $r_{b}=0.6, k_{b}=10, \eta=0.06, \delta=0.5, \alpha=3, \beta=1, r_{v}=3$, and $k_{v}=150$. (a) ET $\in[2,9.07]$; (b) ET = 4; (c) $\mathrm{ET}=8 ;(\mathrm{d}) \mathrm{ET}=5$.

Figure 6(a) shows that there is sliding bifurcation of Filippov system (7) when the ET is set as the different values in [2,9.07]. There is only one stable equilibrium $E_{11}$ of subsystem $S_{1}$ and a stable equilibrium $E_{21}$ of subsystem $S_{2}$. The three cases of Figure $6(\mathrm{a})$ are presented in
Figures $6(\mathrm{~b})-6(\mathrm{~d})$. If $B_{11}>B_{21}>\mathrm{ET}(\mathrm{ET}=4)$, the Filippov system stabilizes at the equilibrium $E_{21}$, which is regular and denoted by $E_{21}^{R}$ (see Figure 6(b)). If $\mathrm{ET}>B_{11}>B_{21}(\mathrm{ET}=8)$, the Filippov system stabilizes at $E_{11} \cdot E_{11}$ is virtual and defined by $E_{11}^{R}$ (see Figure 6(c)). If 


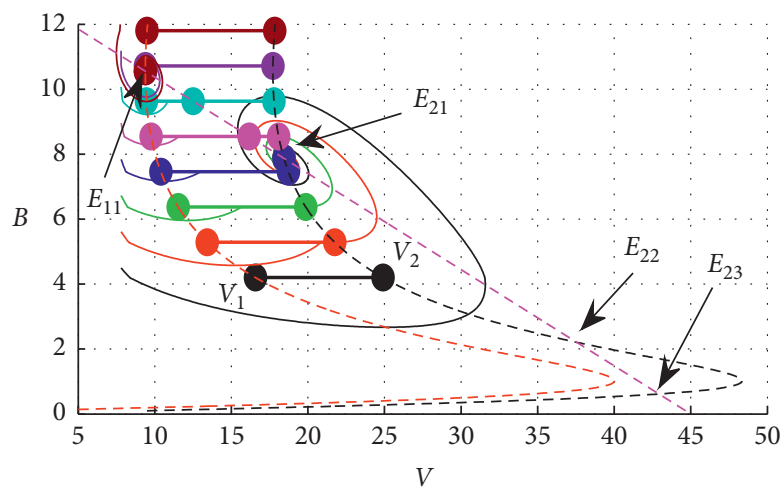

(a)

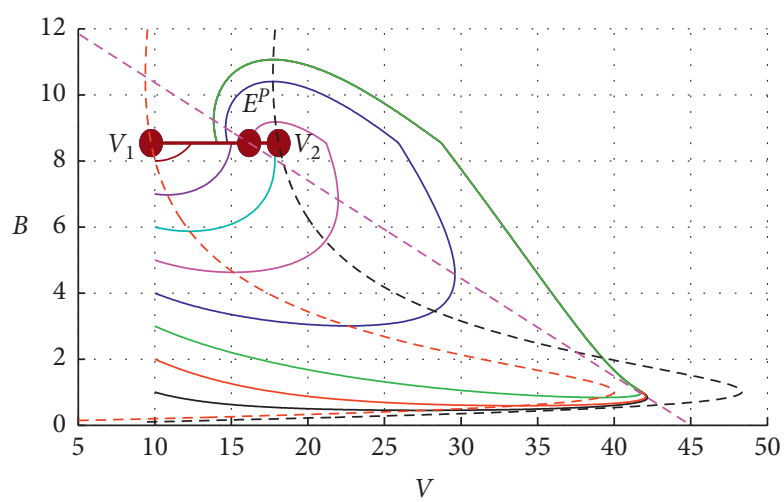

(c)

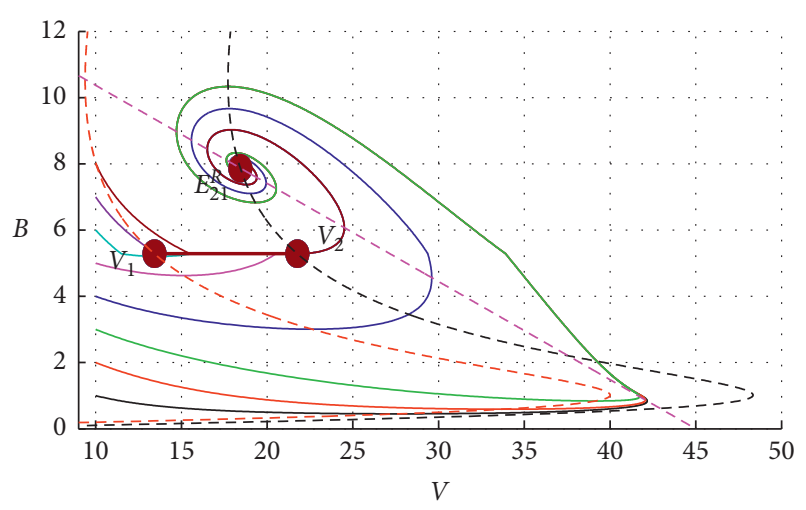

(b)

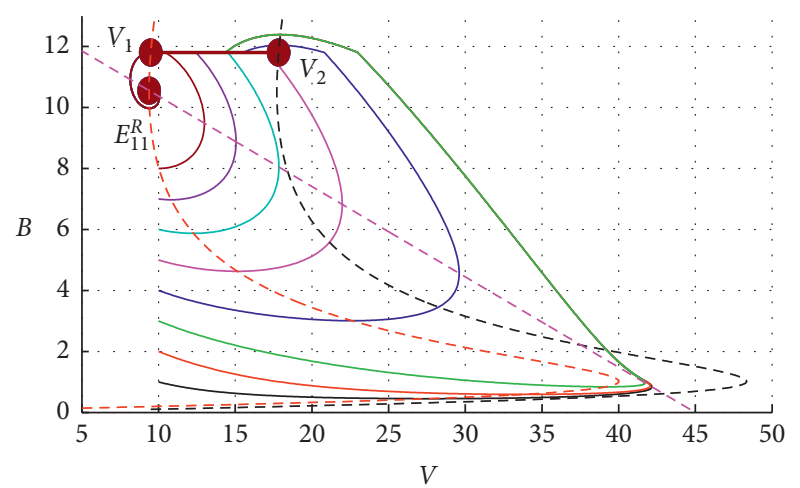

(d)

Figure 7: Sliding bifurcation for Case B.2 of Filippov system (7). Subsystem $S_{1}$ has one stable equilibrium point and subsystem $S_{2}$ is a bistable state. $q_{1}=0.5$ and the other parameters are fixed as $r_{b}=0.5, k_{b}=10, \eta=0.4, \delta=0.15, \alpha=5.7, \beta=1, r_{v}=2$, and $k_{v}=45$. (a) $\mathrm{ET} \in[4.2,11.8]$; (b) $\mathrm{ET}=5.29$; (c) $\mathrm{ET}=8.54$; (d) $\mathrm{ET}=11.8$.

$B_{11}>\mathrm{ET}>B_{21} \quad(\mathrm{ET}=5)$, the Filippov system has a pseudoequilibrium $E^{P}$ and stabilizes on the sliding segment $V_{1} V_{2}$ with $E^{P}$ (see Figure 6(d)). In particular, there exists a pseudoequilibrium on the $E_{11} E_{21}$. While there is no limit cycle in this case, there are no pest outbreaks and the system stabilizes within a relatively small range.

Case B.2: subsystem $S_{2}$ is a bistable state.

Figure 7 (a) shows the sliding bifurcation of the Filippov system for the value of ET varying in the range [4.2, 11.8]. Subsystem $S_{1}$ has only one stable equilibrium $E_{11}$, subsystem $S_{2}$ is a bistable state where $E_{21}$ and $E_{23}$ are stable. Figures $7(\mathrm{~b})-7(\mathrm{~d})$ presents the three cases of Figure 7(a). In Figure 7(b), if $B_{11}>B_{21}>$ ET $>B_{22}>B_{23}$ $(\mathrm{ET}=5.29)$, the Filippov system always stabilizes at $E_{21}$, which is real and denoted by $E_{21}^{R}$. In Figure $7(\mathrm{c})$, if $B_{11}>\mathrm{ET}>B_{21}>B_{22}>B_{23} \quad(\mathrm{ET}=8.54)$, the Filippov system has a pseudoequilibrium $E^{P}$ and stabilizes on the sliding segment $V_{1} V_{2}$ with $E^{P}$. There is a pseudoequilibrium on the $E_{11} E_{21}$. In Figure $7(\mathrm{~d})$, if ET $>B_{11}>B_{21}>B_{22}>B_{23}(\mathrm{ET}=11.8)$, Filippov system (7) is stable at $E_{11}$ with different initial values. $E_{11}$ is regular and denoted by $E_{11}^{R}$. In this case, when $q_{1}=0.5$, the pest population density will stabilize within a relatively large range.
Case B.3: subsystem $S_{2}$ has only one stable refuge equilibrium point.

Figure 8(a) presents the sliding mode phenomena for the different values of ET $(\mathrm{ET} \in[0.1,12])$. Subsystem $S_{1}$ has only one stable equilibrium $E_{11}$ while subsystem $S_{2}$ has only one stable equilibrium $E_{21}$, which is a stable refuge equilibrium point. Figures $8(\mathrm{~b})-8(\mathrm{~d})$ present the three cases of Figure 8(a). In Figure 8(b), if $B_{11}>B_{21}>\mathrm{ET}(\mathrm{ET}=0.1)$, no matter how large the initial values are, the Filippov system stabilizes at $E_{21}$ (i.e., a stable refuge equilibrium point). In Figure 8(c), if $B_{11}>\mathrm{ET}>B_{21}(\mathrm{ET}=6.9)$, the Filippov system has a pseudoequilibrium $E^{P}$ and stabilizes on the $V_{1} V_{2}$ (i.e., the sliding segment) with $E^{P}$. Moreover, $E^{P}$ emerges on the $E_{11} E_{21}$. In Figure $8(\mathrm{~d})$, if $\mathrm{ET}=B_{11}>B_{21}$ $(\mathrm{ET}=10.6)$, the equilibrium $E_{11}$ becomes a stable refuge equilibrium point. The Filippov system stabilizes on the $V_{1} V_{2}$ with $E_{11}$. $E_{11}$ is virtual and denoted by $E_{11}^{V}$. When $q_{1}=0.9$, the pest population density stabilizes within a smaller range than in the case of $q_{1}=0.5$ as shown in Figure 7. This means that the proper strategy can better control pests.

4.3. Case C. Subsystem $S_{1}$ has three equilibrium points, two of which are stable. There are two subcases for subsystem $S_{2}$. 


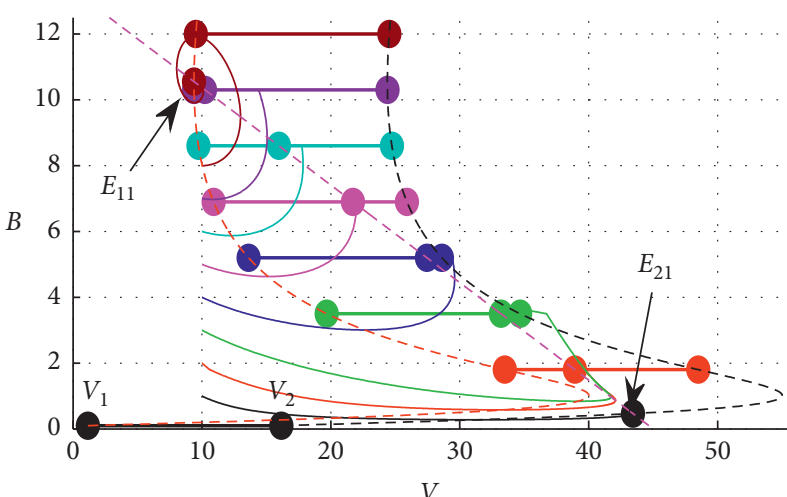

(a)

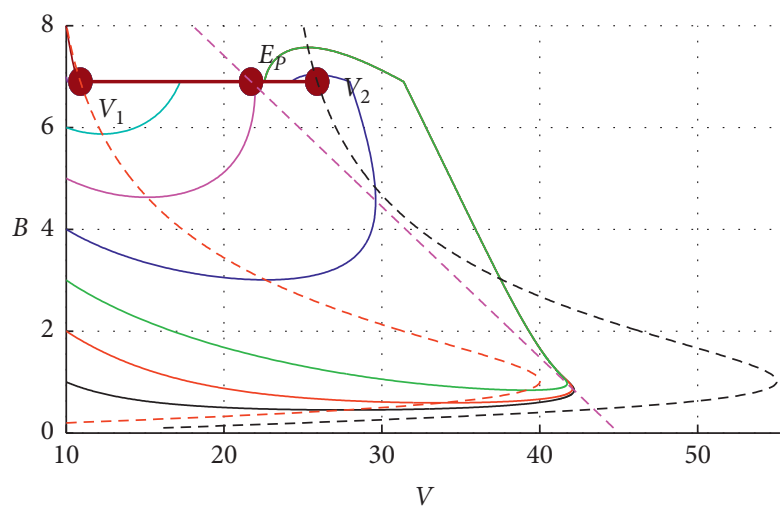

(c)

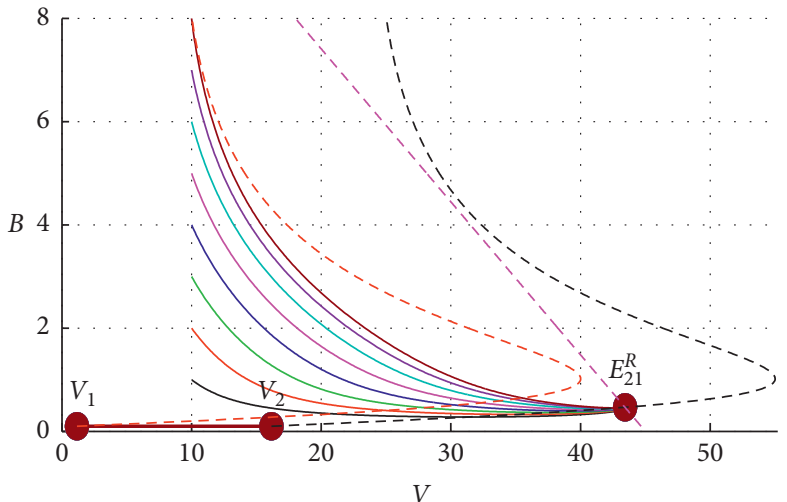

(b)

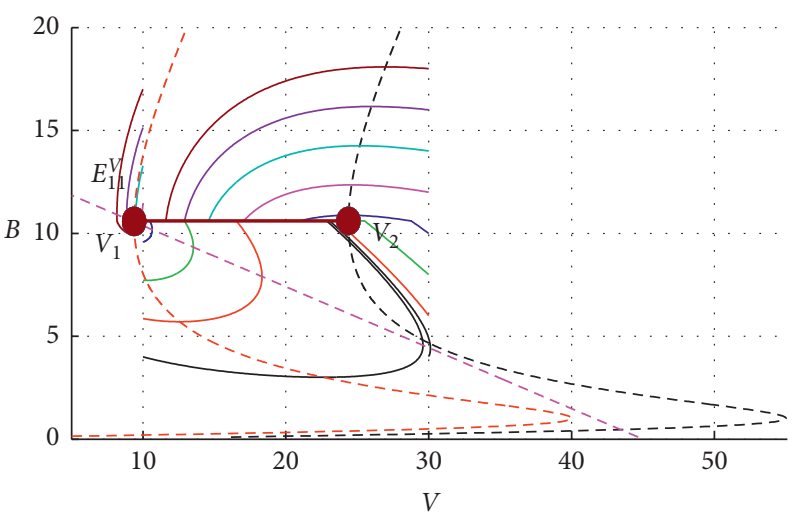

(d)

Figure 8: Sliding bifurcation for Case B.3 of Filippov system (7). Subsystem $S_{1}$ has a stable equilibrium point. Subsystem $S_{2}$ has only one stable refuge equilibrium point. $q_{1}=0.9$, the other parameters are the same as those in Figure 7. (a) ET $\in[0.1,12]$; (b) ET $=0.1 ;(\mathrm{c}) \mathrm{ET}=6.9$; (d) $\mathrm{ET}=10.6$.

(i) Subsystem $S_{2}$ is a bistable state

(ii) Subsystem $S_{2}$ has a stable refuge equilibrium point

The sliding mode phenomena in the two subcases of Case $C$ are discussed as follows:

Case C.1: Subsystem $S_{2}$ is bistable.

Figure 9(a) presents the sliding bifurcation of the Filippov system for the different values of ET in the range $[6.4,13.6]$. Both subsystem $S_{1}$ and subsystem $S_{2}$ have three equilibrium points, namely $E_{11}, E_{12}, E_{13}$, $E_{21}, E_{22}$ and $E_{23} . E_{11}, E_{13}, E_{21}$ and $E_{23}$ are stable. Subsystems $S_{1}$ and $S_{2}$ are bistable states. The three cases of Figure 9(a) are presented in Figures 9(b)-9(d). If ET $>B_{11}>B_{21}>B_{22}>B_{12}>B_{13}>B_{23} \quad(\mathrm{ET}=15)$, the Filippov system stabilizes at $E_{11}$, which is called a regular equilibrium of subsystem $S_{1}$ and denoted by $E_{11}^{R}$ (see Figure 9(b)). If $B_{11}>\mathrm{ET}>B_{21}>B_{22}>B_{12}>$ $B_{13}>B_{23} \quad(\mathrm{ET}=10.5)$, the Filippov system has a pseudoequilibrium $E^{P}$ and stabilizes on the sliding segment $V_{1} V_{2}$ with $E^{P}$ (see Figure 9(c)). If $B_{11}>B_{21}>\mathrm{ET}>B_{22}>B_{13}>B_{23}(\mathrm{ET}=6.4)$, the Filippov system stabilizes at $E_{21}$, which is called a regular equilibrium of subsystem $S_{2}$ and denoted by $E_{21}^{R}$ (see Figure $9(d)$ ). Therefore, in this case, if the density of the pest population is high, the pest population can be well controlled; if the initial values is relatively small, the Filippov system stabilize at a refuge equilibrium point.

Case C.2: Subsystem $S_{2}$ has only one stable refuge equilibrium point.

Figure 10(a) shows the sliding bifurcation of the Filippov system under the different values of ET in the range $[5.3,13.8]$. Subsystem $S_{1}$ has three equilibrium points, namely $E_{11}, E_{12}$ and $E_{13}$. Subsystem $S_{2}$ has an equilibrium $E_{21}$. There is a pseudoequilibrium on the $E_{11} E_{12}$. Figure $10(\mathrm{~b})$ shows that a pseudoequilibrium $E^{P}$ emerges if $B_{11}>\mathrm{ET}>B_{12}>B_{13}>B_{21} \quad(\mathrm{ET}=6.5)$. When the value of $B$ is large, the Filippov system is stable on the $V_{1} V_{2}$ with $E^{P}$. When the value of $B$ is small, the Filippov system stabilizes at a refuge equilibrium point. Therefore, when $q_{1}=0.6$, the pest population density can be controlled in a larger range than in the case of $q_{1}=0.4$ as shown in Figure 9.

4.4. Case D. Subsystem $S_{1}$ and subsystem $S_{2}$ have three equilibrium points. Only one is a stable refuge equilibrium point.

In Figure 11(a), subsystem $S_{1}$ has the three equilibria $E_{11}$, $E_{12}$, and $E_{13} . E_{11}$ and $E_{12}$ are unstable and colored black. Only the red $E_{13}$ is stable. In Figure 11(b), subsystem $S_{1}$ has 


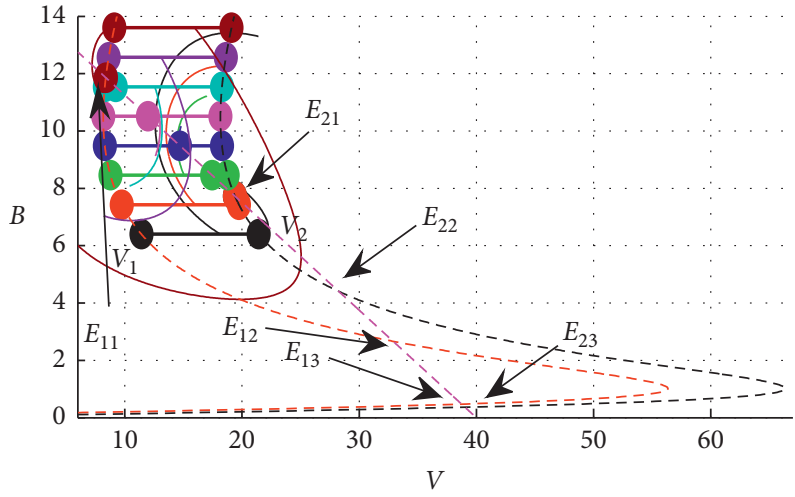

(a)

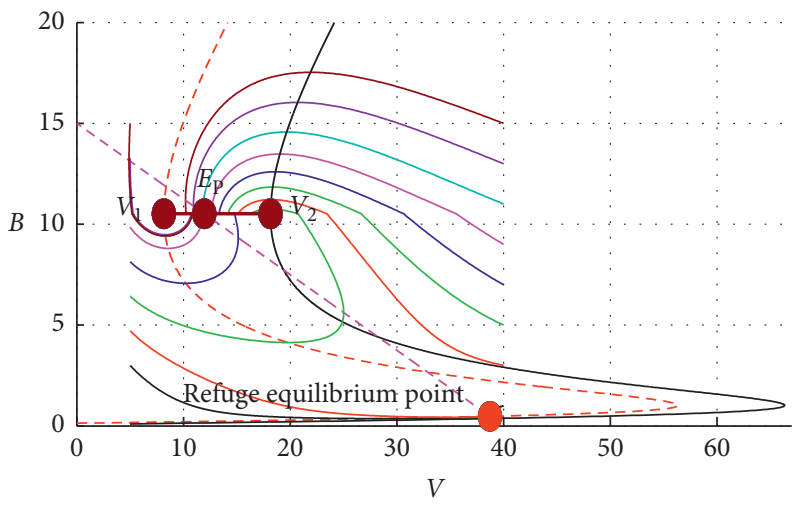

(c)

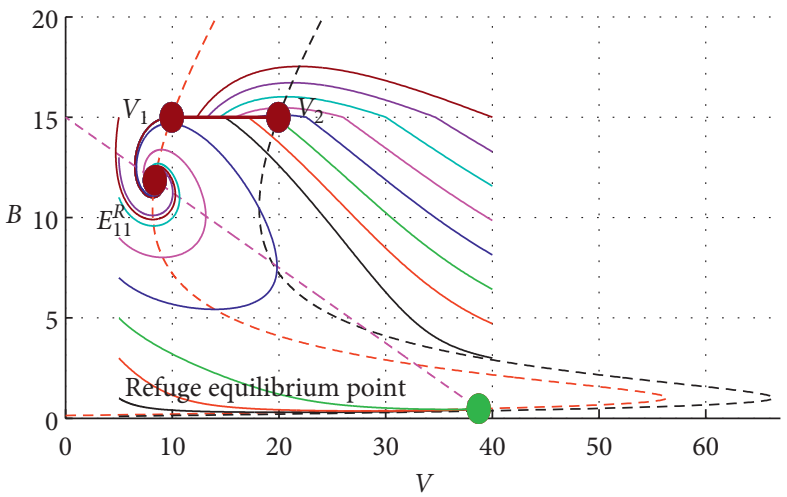

(b)

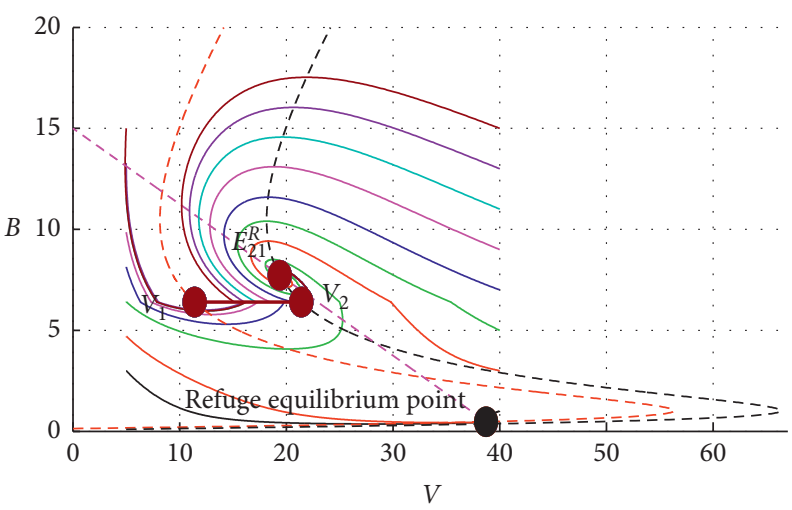

(d)

Figure 9: Sliding bifurcation for Case C.1 of Filippov system (7). Both subsystem $S_{1}$ and subsystem $S_{2}$ are bistable states. $q 1=0.4$, parameters are $r_{b}=0.8, k_{b}=15, \eta=0.4, \delta=0.1, \alpha=6, \beta=1, r_{v}=1.5$, and $k_{v}=40$. (a) ET $\in[6.4,11.6]$; (b) ET = 15; (c) ET = 10.5; (d) $\mathrm{ET}=6.4$.

the three equilibria $E_{21}, E_{22}$ and $E_{23}$. The black $E_{21}$ and $E_{22}$ are unstable, and only the smallest red dot $E_{23}$ is stable. follows.

The sliding mode phenomena of Case D are discussed as

Figure 12(a) shows the sliding bifurcation of the Filippov system for the ET in the range $[3.6,16.4]$. Both subsystem $S_{1}$ and subsystem $S_{2}$ have three equilibrium points. Only one is a stable refuge equilibrium point. Figures 12(b)-12(d) present the three cases of Figure 12(a). If $B_{11}>\mathrm{ET}>$ $B_{21}>B_{22}>B_{12}>B_{13}>B_{23}(\mathrm{ET}=9.1)$, the Filippov system has a pseudoequilibrium $E^{P}$ and stabilizes on the sliding segment $V_{1} V_{2}$ with $E^{P}$ (see Figure 12(b)). If ET $>B_{11}>B_{21}>B_{22}>B_{12}>B_{13}>B_{23} \quad($ ET $=12.7)$, Filippov system (7) has a stable sliding limit cycle and stabilizes on it (see Figure 12(c)). Figure 12(d) has no limit cycle in contrast with Figure 12(c). Therefore, regardless of their initial values, the Filippov system stabilizes at a stable refuge equilibrium point as shown in Figures 12(b)-12(d). In this case, when $q_{1}=0.4$, the pest population remains stable within a relatively large range.

Overall, in Cases A-D, the value of $q_{1}$ is large and the number of beetles is controlled within a certain range that is better. Taking spraying pesticides as an example, from the perspective of economic costs, there is no desire to use more pesticides. In addition, from a biomathematical point of view, the spraying of pesticides (i.e., a chemical measure) is performed frequently to kill beetles, which creates pollution. Therefore, the results show that it is crucial to choose a optimal strategy (or the dosage of pesticide) if the pest density reaches the ET.

\section{Conclusion}

A novel Filippov forest-pest system with TPC concerning IPM was proposed. The system comprises two subsystems $S_{1}$ and $S_{2}$. Our aim is to find the best time and strategy for successful pest control. The conditions for the existence and stability of equilibria of subsystems were addressed. Moreover, the sliding segment was determined. Several types of equilibria of the proposed system were defined, i.e., the regular equilibrium, virtual equilibrium, pseudoequilibrium, boundary equilibrium, and a special point called the tangent point. Meanwhile, nullclines of the Filippov system were also defined, which were related to the equilibria of these two susystems and used to determine their existence. That is, the points of intersection between nullclines were these equilibrium points of the system, and the two endpoints of the sliding segment $\left(V_{1} V_{2}\right)$ were on the nullclines.

Four cases of the sliding bifurcations of the Filippov system with respect to different types of equilibrium points of subsystems were discussed. Details are given in Table 1. In Case A.1, both subsystem $S_{1}$ and subsystem $S_{2}$ had only 


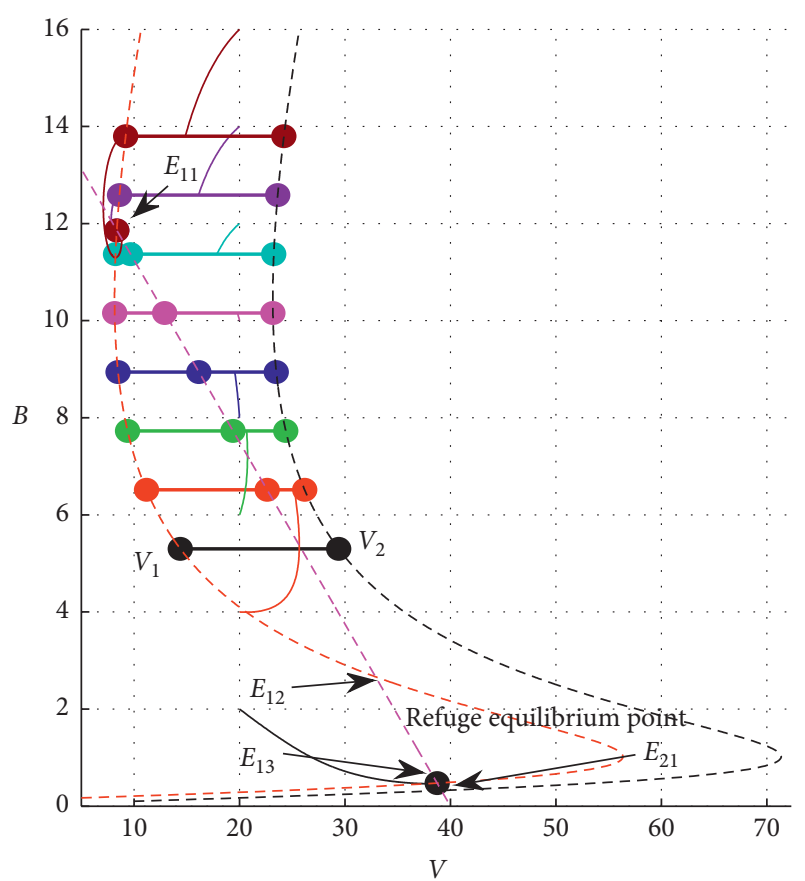

(a)

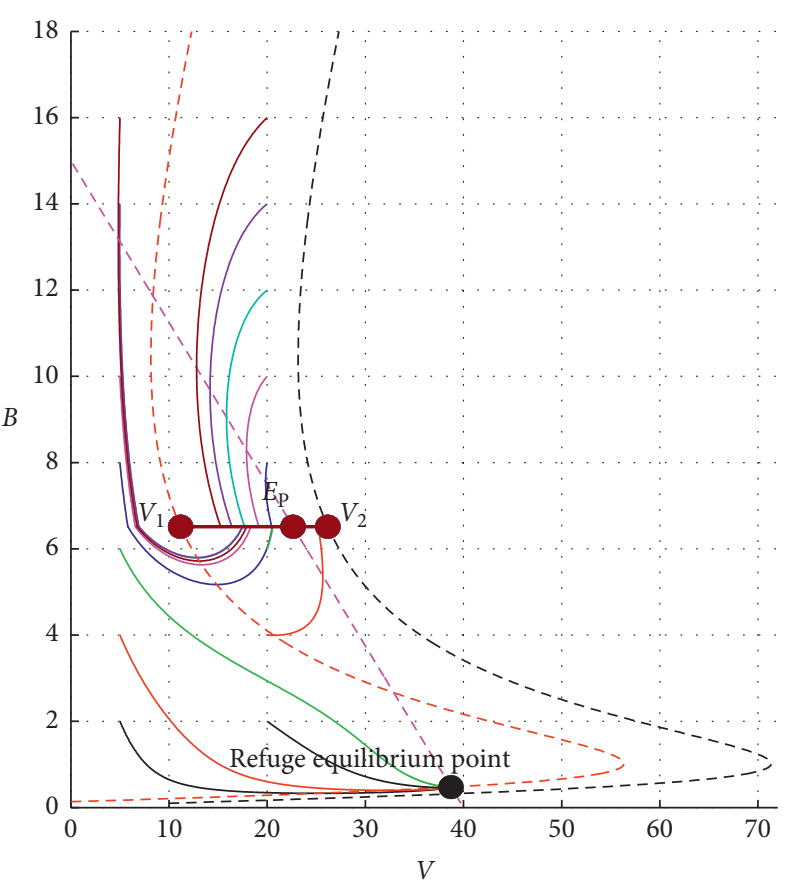

(b)

Figure 10: Sliding bifurcation for Case C.2 of Filippov system (7). Subsystem $S_{2}$ has only one refuge equilibrium point. $q_{1}=0.6$, the other parameters are the same as those in Figure 9. (a) ET $\in[5.3,13.8]$; (b) $\mathrm{ET}=6.5$.

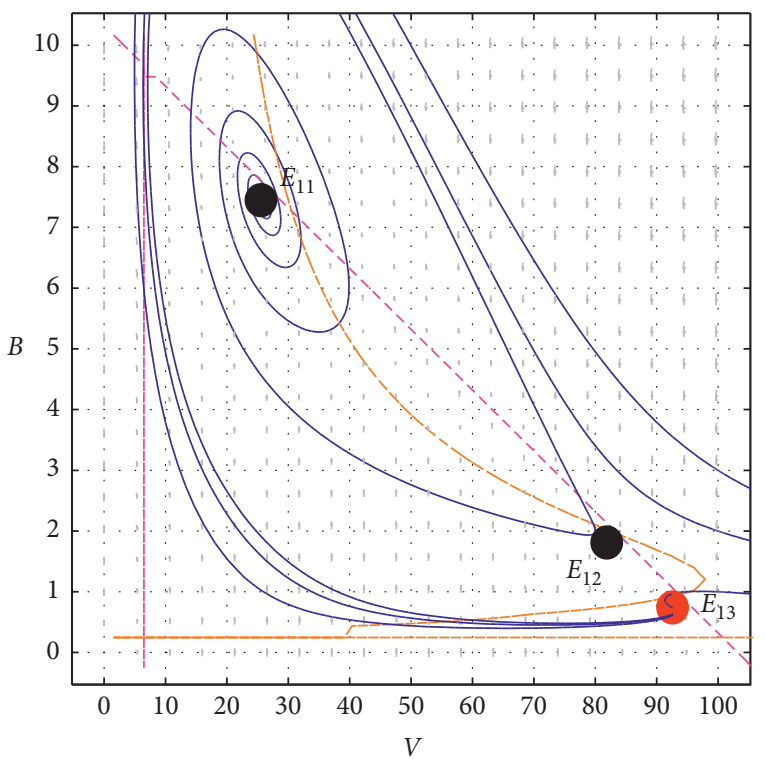

(a)

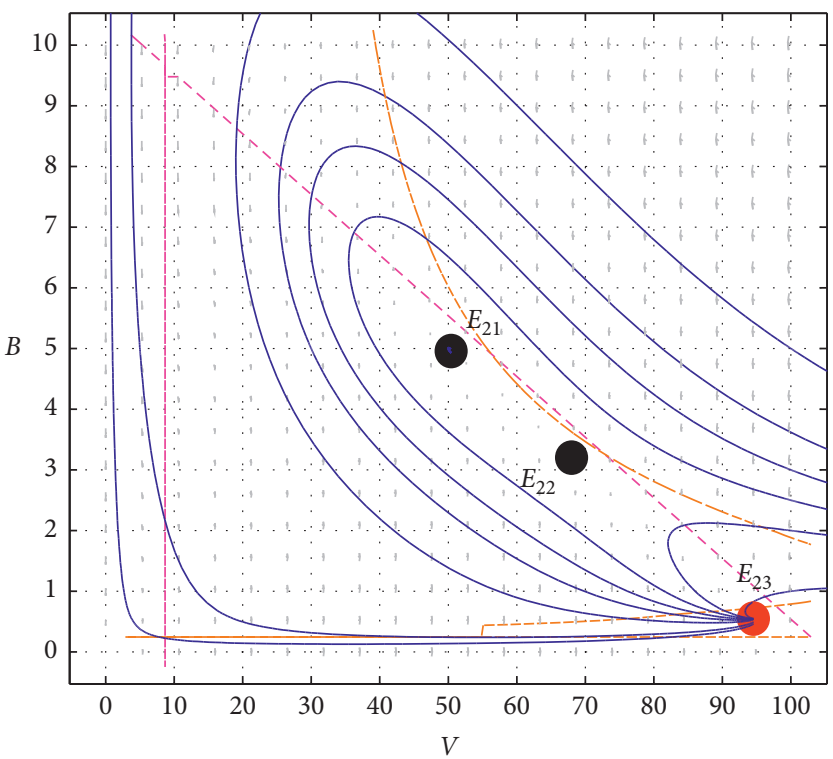

(b)

Figure 11: Existence of equilibria for Case D of Filippov system (7). Parameters are fixed as $r_{b}=0.1, k_{b}=10, \eta=0.15, \sigma=0.2, \alpha=6, \beta=1$, $r_{v}=2$, and $k_{v}=100$. (a) $q_{1}=0$; (b) $q_{1}=0.4$.

an unstable equilibrium point (see Figures 1(a) and 1(b)), the system had limit cycles and stabilized to the larger limit cycle regardless of the initial values ( $V$ and $B$ ) (see Figure 2).

Subsystem $S_{2}$ was a bistable state in Cases A.2, B.2, and C.1 (see Figures 3, 7, and 9). For instance, in Cases A.2 and
B.2, subsystem $S_{1}$ had an unstable equilibrium point (see Figure 1(a)) and subsystem $S_{1}$ had a stable equilibrium point (see Figure 5(a)), subsystem $S_{2}$ had three equilibria (i.e., $E_{21}$, $E_{22}$, and $E_{23}$ ) and equilibria (i.e., $E_{21}$ and $E_{23}$ ) were stable (see Figures 1(c) and 5(c)). In Case C.1, both subsystem $S_{1}$ and subsystem $S_{2}$ are bistable states. 


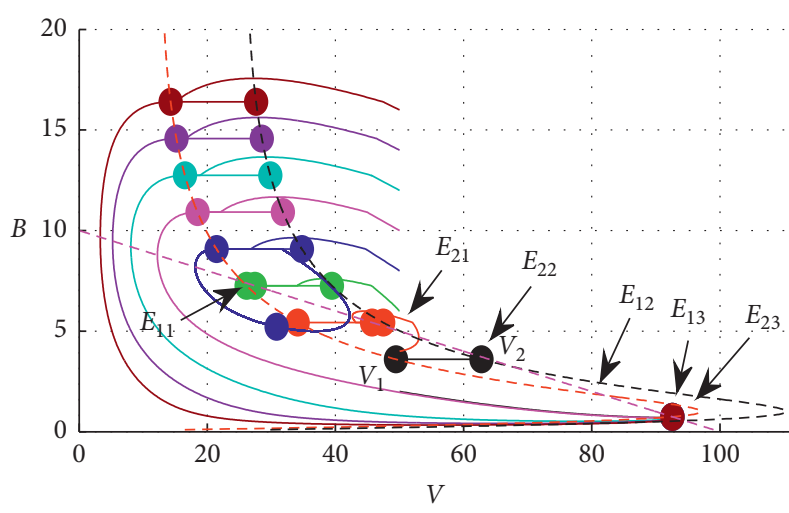

(a)

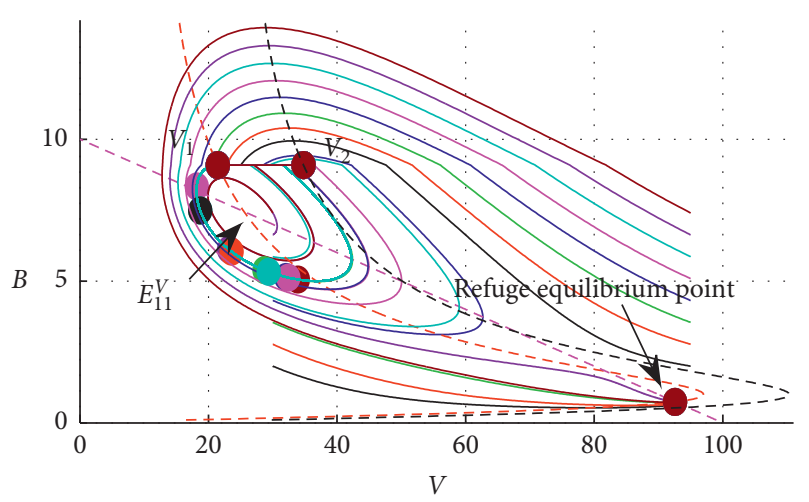

(c)

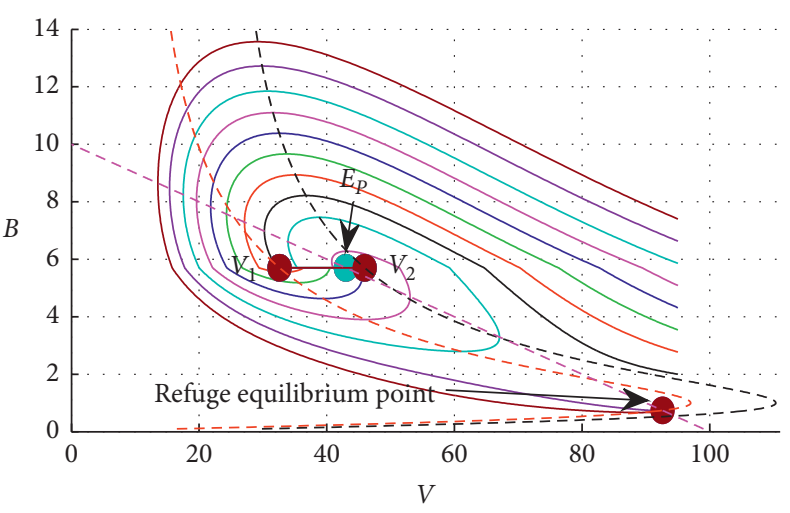

(b)

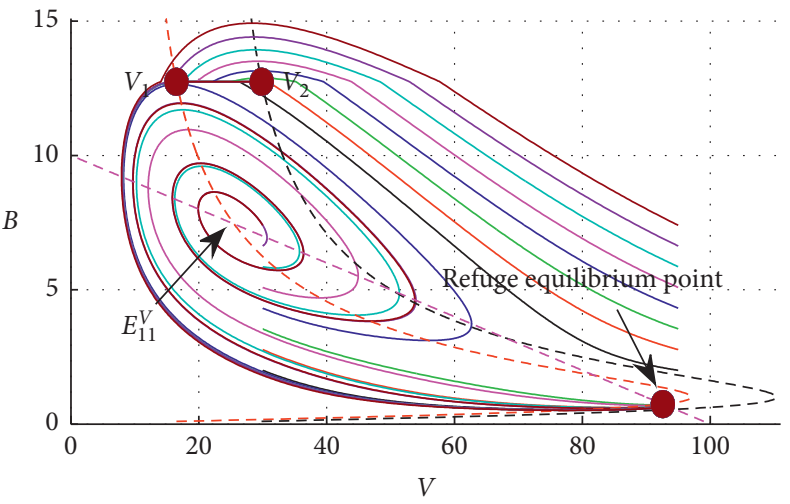

(d)

FIgURE 12: Sliding bifurcation for Case D of Filippov system (7). $q 1=0.4$, the other parameters are the same as those in Figure 11. (a) $\mathrm{ET} \in[3.6,16.4]$; (b) $\mathrm{ET}=5.7$; (c) $\mathrm{ET}=9.1$; (d) $\mathrm{ET}=12.7$.

Subsystem $S_{2}$ had only one stable refuge equilibrium point in Cases A.3, B.3, C.2, and D. For example, in Case A.3, subsystem $S_{1}$ had only an unstable equilibrium point. In Case B.3, subsystem $S_{1}$ had only a stable equilibrium point. When the number of pests and forests was relatively small, subsystem $S_{2}$ had only one stable refuge equilibrium point (see Figures 1(d) and 5(d)), the system was stable at it (see Figures 4 and 8). In Case C.2, subsystem $S_{1}$ was the bistable state and only one stable refuge equilibrium point existed in subsystem $S_{2}$. Moreover, the system stabilized on the $V_{1} V_{2}$ with pseudoequilibrium $E^{\mathrm{P}}$ (see Figure 10). In Case D, both subsystems $S_{1}$ and $S_{2}$ had three equilibrium points (see Figure 11) and only one was a stable refuge equilibrium point. The Filippov system stabilized at a stable refuge equilibrium point with $\mathrm{ET}$ in the range $[3.6,16.4]$ (see Figure 12). A stable equilibrium point appeared in subsystem $S_{1}$ and subsystem $S_{2}$ (see Figures 5(a) and 5(b)). Especially, in Case A, the stable limit cycles existed in subsystem $S_{1}$.

In addition, it was verified that the pseudoequilibrium was the point of intersection of the sliding segment and nullclines of the Filippov system, and the pseudoequilibrium existed on the $V_{1} V_{2}$. In Case A, when $q_{1}=0.45$, it was the most suitable for controlling pests. In Case $\mathrm{B}$, the results revealed that the proper use of pesticides can effectively control pests. In Case C, when $q_{1}=0.6$, the effects of pest control were the best. Therefore, to some extent, the more the pesticides were used, the better the number of beetles will be controlled within a certain range so that the aim of IPM strategies was achieved.

In summary, the Filippov system had sliding limit cycles, a bistable state, and a stable refuge equilibrium point by means of sliding bifurcation analysis. From the perspective of practical biological significance, when the pest density reached the ET, the optimal strategy should be used, namely, the proper number of pests was captured, transferred, or killed by using the cultural and chemical strategies (i.e., the right amount of pesticide needed to be sprayed). From an economic point of view, it was beneficial to reduce the use of manpower, material resources, and financial resources and protected the environment. In the future, different control policies in which the threshold is also a linear or nonlinear function about forests and pests deserve investigation.

\section{Data Availability}

No data were used to support this study. In our study, there are only some numerical simulations to support our main result, and some parameter values to support the result of this paper are included within the article. 


\section{Conflicts of Interest}

The authors declare that there are no conflicts of interest regarding the publication of this paper.

\section{Acknowledgments}

This work was supported in part by the National Natural Science Foundation of China under grants 11761031, 61562025 and 11961024; in part by the Hubei Technical Innovation Special Project (Key Project) of China under grant 2018AKB035; and in part by the Special Funds for "Double First-Class" Construction in Hubei Province. The authors thank Glenn Pennycook, MSc, from Liwen Bianji, Edanz Group China (http://www.liwenbianji.cn/ac), for editing the English text of a draft of this manuscript.

\section{References}

[1] C. Sims, D. Aadland, D. Finnoff, and J. Powell, "How ecosystem service provision can increase forest mortality from insect outbreaks," Land Economics, vol. 89, no. 1, pp. 154-176, 2013.

[2] K. F. Raffa, B. H. Aukema, B. J. Bentz et al., "Cross-scale drivers of natural disturbances prone to anthropogenic amplification: the dynamics of bark beetle eruptions," Bioscience, vol. 58, no. 6, pp. 501-517, 2008.

[3] H. K. Preisler, A. A. Ager, and J. L. Hayes, "Probabilistic risk models for multiple disturbances: an example of forest insects and wildfires," in Advances in Threat Assessment and Their Application to Forest and Rangeland Management, J. M. Pye, H. M. Rauscher, Y. Sands et al., Eds., vol. 802, pp. 371-379, US Department of Agriculture, Forest Service, Pacific Northwest and Southern Research Stations: 371-379, Portland, OR, USA, 2010, Gen. Tech. Rep. PNW-GTR-802.

[4] B. Chen-Charpentier and M. C. A. Leite, "A model for coupling fire and insect outbreak in forests," Ecological Modelling, vol. 286, no. 4, pp. 26-36, 2014.

[5] S. Liu, B. Bond-Lamberty, J. A. Hicke et al., "Simulating the impacts of disturbances on forest carbon cycling in North America: processes, data, models, and challenges," Journal of Geophysical Research Biogeosciences, vol. 116, no. 4, pp. 127134, 2011.

[6] M. C. A. Leite, B. Chen-Charpentier, and F. B. Agusto, "A mathematical model of tree harvesting in age-structured forests subject to beetle infestations," Computational and Applied Mathematics, vol. 37, no. 3, pp. 3365-3384, 2018.

[7] M. Leite, B. Chen-Charpentier, and F. B. Agusto, "Maximizing tree harvesting benefit from forests under insect infestation disturbances," PLoS One, vol. 13, no. 8, Article ID e0200575, 2018.

[8] H. J. Barclay, T. Schivatcheva, and C. Li, "Equilibrium forest age structure: simulated effects of random wild fires, fire control, and harvesting," Journal of Ecosystems and Management, vol. 10, no. 2, pp. 108-114, 2009.

[9] M. Ducey, J. Gunn, and A. Whitman, "Late-successional and old-growth forests in the northeastern United States: structure, dynamics, and prospects for restoration," Forests, vol. 4, no. 4, pp. 1055-1086, 2013.

[10] C. R. Keyes, T. E. Perry, E. K. Sutherland, D. K. Wright, and J. M. Egan, "Variable-retention harvesting as a silvicultural option for lodgepole pine," Journal of Forestry, vol. 112, no. 5, pp. 440-445, 2014.
[11] A. F. Filippov, Differential Equations with Discontinuous Righthand Sides, Kluwer, Dordrecht, Netherlands, 1988.

[12] M. Bernardo, C. Budd, A. R. Champneys, and P. Kowalczyk, Piecewise-smooth Dynamical Systems: Theory and Applications, Springer Science \& Business Media, Berlin, Germany, 2008.

[13] Y. Yang, X. Liao, and T. Dong, "Period-adding bifurcation and chaos in a hybrid hindmarsh-rose model," Neural Networks, vol. 105, pp. 26-35, 2018.

[14] J. Yang, G. Tang, and S. Tang, "Modelling the regulatory system of a chemostat model with a threshold window," Mathematics and Computers in Simulation, vol. 132, pp. 220-235, 2017.

[15] C. Chen, Y. Kang, and R. Smith, "Sliding motion and global dynamics of a filippov fire-blight model with economic thresholds," Nonlinear Analysis: Real World Applications, vol. 39, pp. 492-519, 2018.

[16] S. Tang, J. Liang, Y. Xiao, and R. A. Cheke, "Sliding bifurcations of filippov two stage pest control models with economic thresholds," SIAM Journal on Applied Mathematics, vol. 72, no. 4, pp. 1061-1080, 2012.

[17] Y. Yang and X. Liao, "Filippov hindmarsh-rose neuronal model with threshold policy control," IEEE Transactions on Neural Networks and Learning Systems, vol. 30, no. 1, pp. 306-311, 2019.

[18] A. Wang, Y. Xiao, and H. Zhu, "Dynamics of a filippov epidemic model with limited hospital beds," Mathematical Biosciences \& Engineering, vol. 15, no. 3, pp. 739-764, 2018.

[19] X. Zhang and S. Tang, "Existence of multiple sliding segments and bifurcation analysis of Filippov prey-predator model," Applied Mathematics and Computation, vol. 239, no. 2, pp. 265-284, 2014.

[20] F. Dercole, A. Gragnani, and S. Rinaldi, "Bifurcation analysis of piecewise smooth ecological models," Theoretical Population Biology, vol. 72, no. 2, pp. 197-213, 2007.

[21] W. Qin, X. Tan, M. Tosato, and X. Liu, "Threshold control strategy for a non-smooth filippov ecosystem with group defense," Applied Mathematics and Computation, vol. 362, p. 124532, 2019.

[22] L. Liu, C. Xiang, and G. Tang, "Dynamics analysis of periodically forced filippov holling ii prey-predator model with integrated pest control," IEEE Access, vol. 7, pp. 113889113900, 2019.

[23] X. Tan, W. Qin, X. Liu, J. Yang, and S. Jiang, "Sliding bifurcation analysis and global dynamics for a filippov predatorprey system," Journal of Nonlinear Sciences and Applications, vol. 09, no. 06, pp. 3948-3961, 2016.

[24] R. Qu and S. Li, "Attractor and vector structure analyses of bursting oscillation with sliding bifurcation in filippov systems," Shock and Vibration, vol. 2019, Article ID 8213808, 10 pages, 2019.

[25] Y. A. Kuznetsov, S. Rinaldi, and A. Gragnani, "One-parameter bifurcations in planar filippov systems," International Journal of Bifurcation and Chaos, vol. 13, no. 8, pp. 2157-2188, 2003.

[26] C. S. Holling, "The components of predation as revealed by a study of small-mammal predation of the European pine sawfly," The Canadian Entomologist, vol. 91, no. 5, pp. 293-320, 1959.

[27] K. Yang and E. Beretta, "Global qualitative analysis of a ratiodependent predator-prey system," Journal of Mathematical Biology, vol. 36, no. 4, pp. 389-406, 1998.

[28] M. P. Hassell and G. C. Varley, "New inductive population model for insect parasites and its bearing on biological control," Nature, vol. 223, no. 5211, pp. 1133-1137, 1969. 
[29] C. Xiang, Z. Xiang, S. Tang, and J. Wu, "Discrete switching host-parasitoid models with integrated pest control," International Journal of Bifurcation and Chaos, vol. 24, no. 9, p. 1450114, 2014

[30] C. Xiang, Z. Xiang, and Y. Yang, "Dynamic complexity of a switched host-parasitoid model with beverton-holt growth concerning integrated pest management," Journal of Applied Mathematics, vol. 2014, Article ID 501423, 10 pages, 2014.

[31] C. Xiang, Y. Yang, Z. Xiang, and W. Qin, "Numerical analysis of discrete switching prey-predator model for integrated pest management," Discrete Dynamics in Nature and Society, vol. 2016, Article ID 8627613, 11 pages, 2016.

[32] K. Sun, T. Zhang, and Y. Tian, "Dynamics analysis and control optimization of a pest management predator-prey model with an integrated control strategy," Applied Mathematics and Computation, vol. 292, pp. 253-271, 2017.

[33] F. Al Basir, A. M. Elaiw, and S. Ray, "Effect of time delay in controlling crop pest using farming awareness," International Journal of Applied and Computational Mathematics, vol. 5, no. 4, p. 110, 2019.

[34] J. Chowdhury, F. Al Basir, Y. Takeuchi, M. Ghosh, and P. K. Roy, "A mathematical model for pest management in Jatropha curcas with integrated pesticides-an optimal control approach," Ecological Complexity, vol. 37, pp. 24-31, 2019.

[35] F. A. Basir, A. Banerjee, and S. Ray, "Role of farming awareness in crop pest management-a mathematical model," Journal of Theoretical Biology, vol. 461, pp. 59-67, 2019.

[36] V. M. Stern, R. F. Smith, R. van den Bosch, and K. S. Hagen, "The integration of chemical and biological control of the spotted alfalfa aphid: the integrated control concept," Hilgardia, vol. 29, no. 2, pp. 81-101, 1959.

[37] S. Tang, Y. Xiao, and R. A. Cheke, "Multiple attractors of hostparasitoid models with integrated pest management strategies: eradication, persistence and outbreak," Theoretical Population Biology, vol. 73, no. 2, pp. 181-197, 2008.

[38] X. Zhang and S. Tang, "Filippov ratio-dependent preypredator model with threshold policy control," Abstract and Applied Analysis, vol. 2013, Article ID 280945, 13 pages, 2013.

[39] M. I. D. S. Costa and L. D. B. Faria, "Integrated pest management: theoretical insights from a threshold policy," Neotropical Entomology, vol. 39, no. 1, pp. 1-8, 2010.

[40] M. I. D. S. Costa and M. E. M. Meza, "Application of a threshold policy in the management of multispecies fisheries and predator culling," Mathematical Medicine \& Biology: A Journal of the IMA, vol. 23, no. 1, pp. 63-75, 2006.

[41] M. I. D. S. Costa, "Harvesting induced fluctuations: insights from a threshold management policy," Mathematical Biosciences, vol. 205, no. 1, pp. 77-82, 2007.

[42] W. Qin, X. Tan, X. Shi, J. Chen, and X. Liu, "Dynamics and bifurcation analysis of a filippov predator-prey ecosystem in a seasonally fluctuating environment," International Journal of Bifurcation and Chaos, vol. 29, no. 2, Article ID 1950020, 2019.

[43] Y. Xiao and F. V. D. Bosch, "The dynamics of an eco-epidemic model with biological control," Ecological Modelling, vol. 168, no. 1-2, pp. 203-214, 2003.

[44] J. Liang and S. Tang, "Optimal dosage and economic threshold of multiple pesticide applications for pest control," Mathematical \& Computer Modelling, vol. 51, no. 5-6, pp. 487-503, 2010.

[45] J. Liang, S. Tang, R. A. Cheke, and J. Wu, “Adaptive release of natural enemies in a pest-natural enemy system with pesticide resistance," Bulletin of Mathematical Biology, vol. 75, no. 11, pp. 2167-2195, 2013.
[46] A. A. Berryman, "Dynamics of bark beetle populations: analysis of dispersal and redistribution," Bulletin of the Entomological Society, vol. 52, pp. 227-234, 1979.

[47] C. J. MacQuarrie and B. J. Cooke, "Density-dependent population dynamics of mountain pine beetle in thinned and unthinned stands," Canadian Journal of Forest Research, vol. 41, no. 5, pp. 1031-1046, 2011.

[48] K. F. Raffa and A. A. Berryman, "A mechanistic computer model of mountain pine beetle populations interacting with lodgepole pine stands and its implications for forest managers," Forest Science, vol. 32, no. 3, pp. 789-805, 1986.

[49] C. S. Holling, "Some characteristics of simple types of predation and parasitism," The Canadian Entomologist, vol. 91, no. 7, pp. 385-398, 1959.

[50] R. Arditi and L. R. Ginzburg, "Coupling in predator-prey dynamics: ratio dependence," Journal of Theoretical Biology, vol. 139, no. 3, pp. 311-326, 1989.

[51] V. Utkin, J. Guldner, and J. Shi, Sliding Mode Control in Electro-Mechanical Systems, CRC Press, Boca Raton, FL, USA, 2009.

[52] V. I. Utkin, Sliding Modes and Their Applications in Variable Structure Systems, Mir Publishers, Moscow, Russia, 1978.

[53] V. I. Utkin, Sliding Modes in Control and Optimization, Springer Berlin Heidelberg, Berlin, Germany, 1992.

[54] M. D. Bernardo, C. J. Budd, A. R. Champneys et al., "Bifurcations in nonsmooth dynamical systems," SIAM Review, vol. 50, no. 4, pp. 629-701, 2008.

[55] M. Guardia, T. M. Seara, and M. A. Teixeira, "Generic bifurcations of low codimension of planar filippov systems," Journal of Differential Equations, vol. 250, no. 4, pp. 19672023, 2011. 


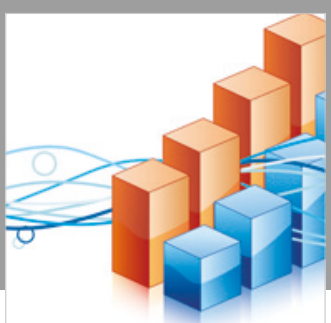

Advances in

Operations Research

\section{-n-m}
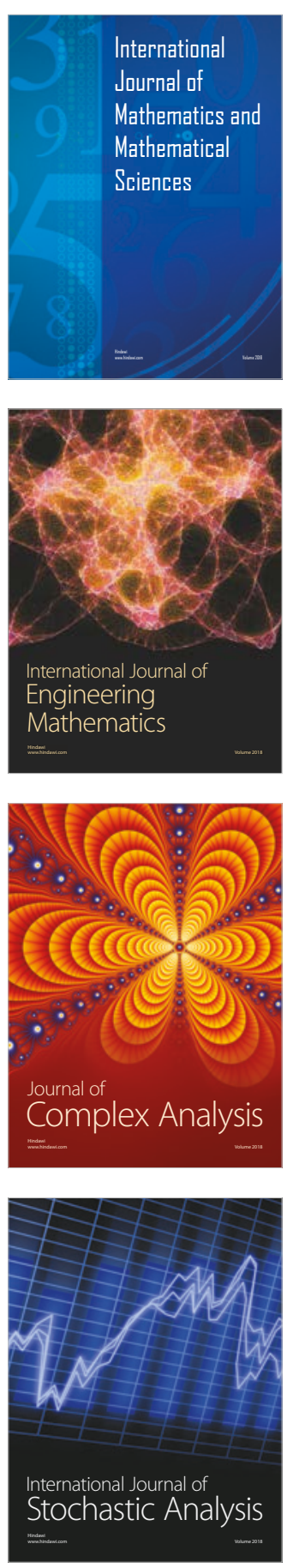
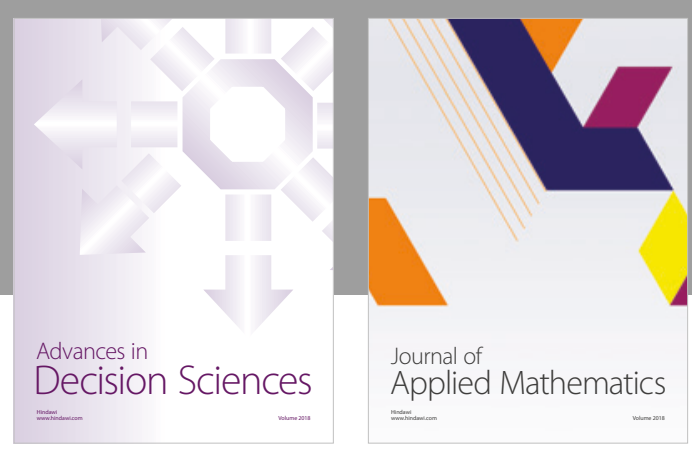

Journal of

Applied Mathematics
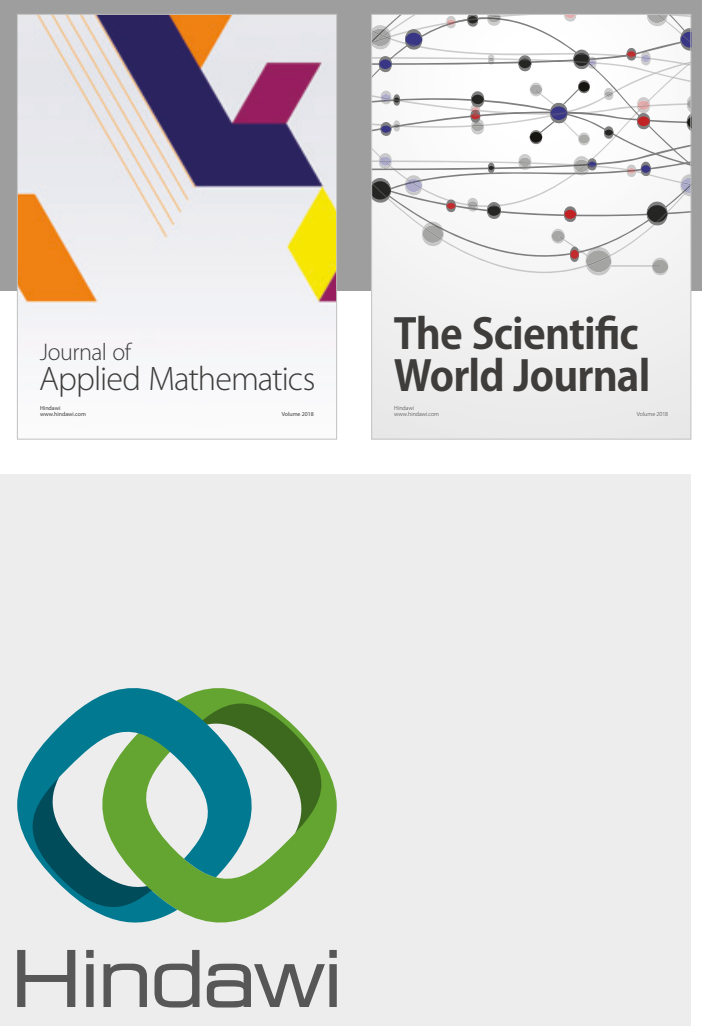

Submit your manuscripts at

www.hindawi.com

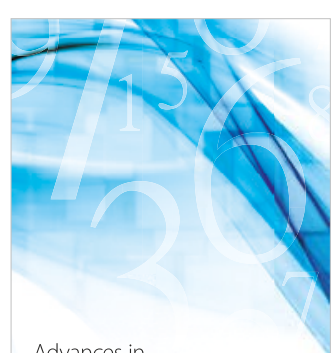

Advances in
Numerical Analysis
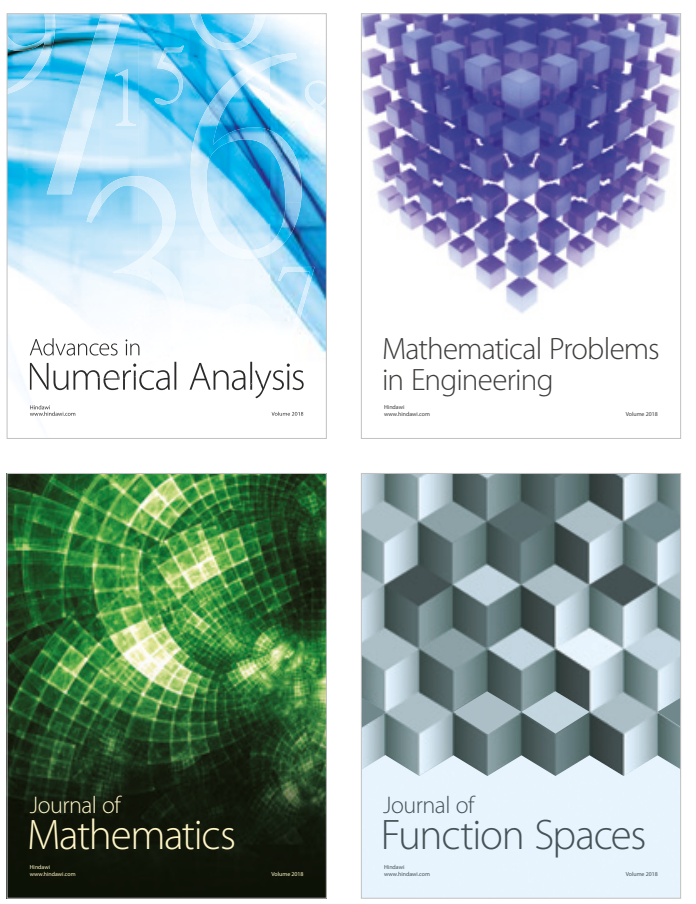

Mathematical Problems in Engineering

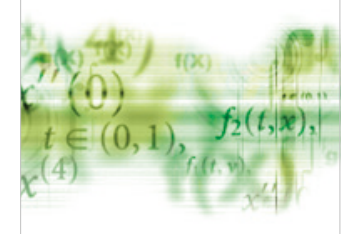

International Journal of

Differential Equations

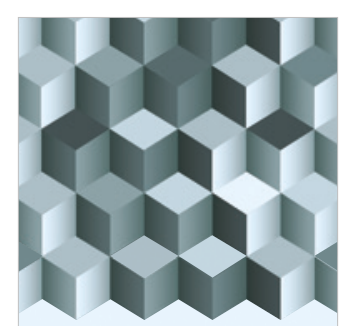

Journal of

Function Spaces
The Scientific

World Journal

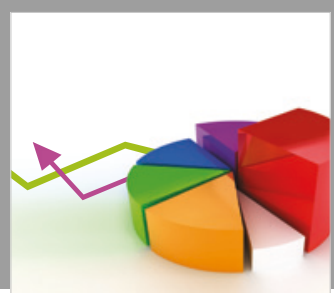

Journal of

Probability and Statistics
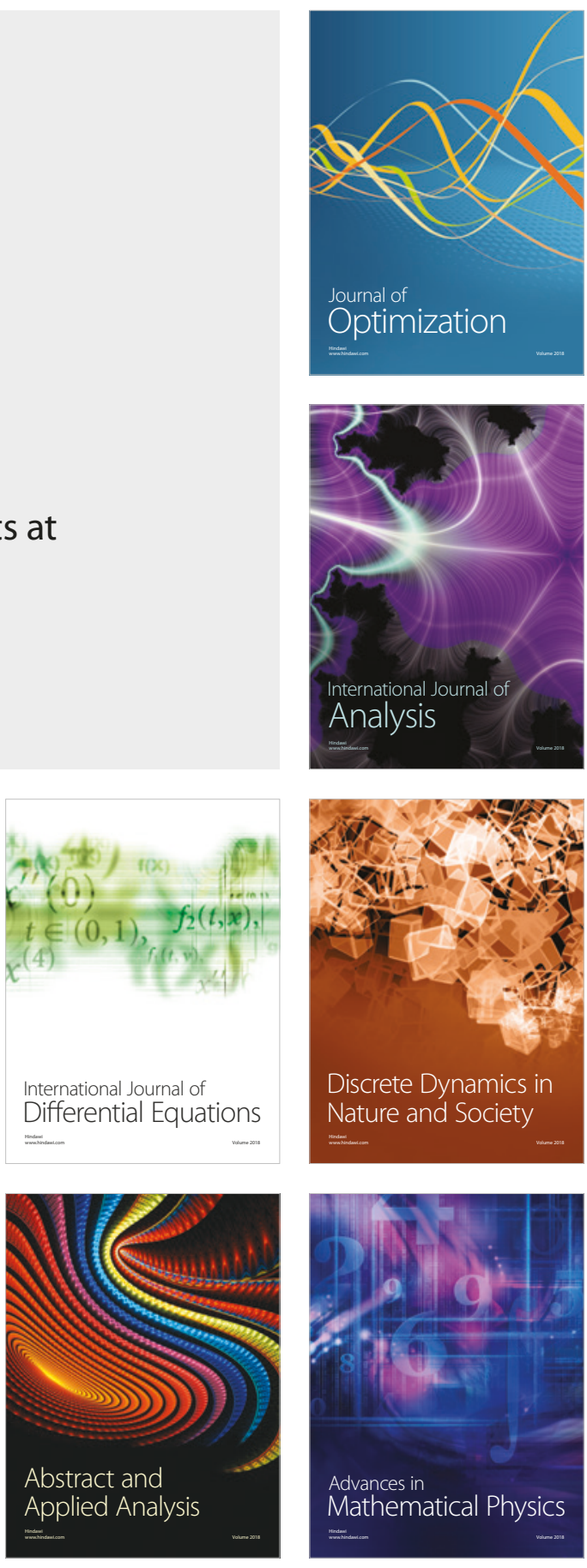